\title{
Electromagnetic fields at the sea bottom induced by a line of immersed electric dipoles
}

\author{
EDSON E.S. SAMPAIO \\ CPGG, Instituto de Geociências, Universidade Federal da Bahia, \# 213-B, \\ Campus Universitário de Ondina, 40170-290 Salvador, BA, Brasil \\ Manuscript received on June 19, 2009; accepted for publication on December 1, 2010
}

\begin{abstract}
The analysis of electromagnetic fields caused by alternate or transient electric currents flowing along a cable in sea water has several applications. It supports the interpretation of electromagnetic geophysical data and safety procedures against the threat of sea mines. The approach to the problem employs a magnetic vector potential in the frequency domain due to a pulse source electric dipole, and performs Laplace and Hankel transforms and integration along the cable, to describe the variation of the magnetic induction field due to an electric dipole of finite length. The result is applicable to shallow or deep sea water environments, adaptable to any transmitting current waveform and useful for wave-field separation. The prospects relate to a horizontal receiving coil at the sea bottom and simulate: a minesweeper campaign with a current source at the sea surface or a geophysical survey with a current source close to the sea floor. Therefore, the present analysis may serve: to define parameters in counter-sweeping of submarine mines; to map the conductivity of sediments under shallow waters for the prevention and control of contamination; and as a first approach in the characterization of offshore mineral and oil economic deposits.
\end{abstract}

Key words: dipoles, electromagnetic energy, induced fields, sea.

\section{INTRODUCTION}

Magnetic tail is a nautical designation for a line of electric dipoles. It consists of a steady state or a transient electric current flowing along a cable of finite length. The tail may be placed at any point between the surface and the bottom of a salty water layer to originate an electromagnetic field. The description of the magnetic field vector in the time domain caused by the tail inside the same region is useful for several applications in physics and in electrical and mechanical engineering to calibrate laboratory simulations and field experiments.

In naval engineering, it is employed to protect ships from the threat of sea mines, for the safety of maritime transportation (Pinheiro and Sampaio 1993, Rayner 2007). In geophysics, it can be of help

E-mail: edson@cpgg.ufba.br 
in investigating the submarine substratum (Constable and Cox 1996, Flosadóttir and Constable 1996, Goldman 1990); to map pollution of the sea bottom in shallow waters (Cheesman et al. 1987, Scholl and Edwards 2007, Souza and Sampaio 2001); to prospect for minerals in the seafloor (Wolfgram et al. 1986, Wynn 1988); and to detect the presence of gas hydrate deposits (Edwards 1997). It is also becoming increasingly important for the prospection and monitoring of offshore hydrocarbon reservoirs (Ellingsrud et al. 2002, Eidesmo et al. 2002).

We compute and analyze in this paper both the spatial and the time variation of the vertical component of the magnetic field due to a magnetic tail for two cases: (1) a current source at the sea surface and a horizontal coil at the sea bottom; and (2) a current source and a horizontal coil at the sea bottom. The first case simulates the situation of a minesweeper campaign and may have application for monitoring pollution in shallow sea waters. The second case simulates a submarine geophysical investigation.

There is a substantial body of geophysical investigation about electromagnetic sources on the sea surface and in the sea floor, as for example: (Chave and Cox 1982, Chave et al. 1991, Constable 2006, Constable and Weiss 2006, Løseth and Ursin 2007, Guimarães and Sampaio 2008a, b). However, this paper has two distinct goals and employs a different procedure from these investigations.

The goals of this paper are: (1) to provide a general solution of the problem, which is valid for any current waveform; and (2) to represent the main part of the wave field as a series of real terms identified with successive reflections at the top and the bottom of a sea water layer confined between the air and a homogeneous substratum. This one-dimensional model represents a new procedure applicable in marine transportation safety and for mapping seafloor pollution of marshes and bays in shallow waters. Though it has a restricted application in geophysics, especially in reservoir characterization, the analysis of 2-D and 3-D scattering problems usually employs the result of a one-dimensional model in building up the respective 2-D or 3-D solutions. Such is the case, for instance, of the Sampaio approximation (Sampaio and Fokkema 1992, Sampaio and Popov 1997, Batista and Sampaio 2003), as well as of the Born or integral equation approximations (Wannamaker et al. 1984, Torres-Verdín and Bostick Jr. 1992, Spies and Habashy 1995, Torres-Verdín and Habashy 2001, Tseng et al. 2003). Furthermore, the contribution of a homogeneous or a layered half-space is usually larger than the contribution scattered by 2-D or 3-D inhomogeneities. So, it is necessary to understand better the homogeneous or 1-D response in order to interpret adequately the inhomogeneity response.

We solve the problem in the time domain by employing the following procedure. First we determine the frequency domain vector potential due to an electric dipole whose current waveform is a delta function in the time domain in free-space. This step is capable of providing the general solution for an arbitrary current waveform by convolving it with the solution for the delta function. Next, we apply the pertinent boundary conditions and solve the correspondent frequency domain problem for the magnetic induction field under quasi-static conditions in a three layered space: air, sea, and substratum. In the following steps, we expand the reflection coefficient and retain only three first order terms, substitute the complex inverse Fourier transform of the delta function by the time derivative of the inverse Laplace transform of the step function, perform a Hankel transform, and integrate along the length of the tail. By doing so, we fulfil the second aim. 


\section{DEVELOPMENT OF THE SOLUTION}

We will develop the algebra of the solution in this section. However, we will not reproduce here the basic details of the mathematical development of this problem because its theory is well known in the geophysical literature (Stratton 1941, Wait 1982, Ward and Hohmann 1988, Pinheiro and Sampaio 1993).

\section{Dipole in a Conductive Infinite Medium}

Let an electric dipole be in a conductive infinite medium as shown in Figure 1. The magnetic vector potential, $\mathbf{A}(x, y, z, \omega)$, and the magnetic induction field, $\mathbf{B}(x, y, z, \omega)$, are related by the following equation:

$$
\mathbf{B}=\nabla \times \mathbf{A},
$$

where: $(x, y, z)$ represent the coordinates of the observation point, $\omega=2 \pi f$, and $f$ is the frequency.

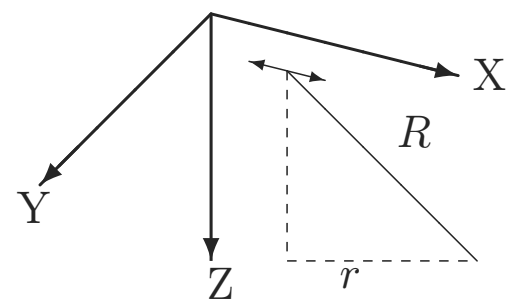

Fig. 1 - A $x$-directed electric dipole $I d x_{0} \mathbf{i}$ at $\left(x_{0}, y_{0}, z_{0}\right)$ in a homogeneous isotropic and infinite medium. Observation point at $(x, y, z) . R$ represents the source-receiver distance, and $r$ the horizontal projection of $R$.

The magnetic vector potential obeys the non-homogeneous Helmholtz wave equation:

$$
\nabla^{2} \mathbf{A}+\kappa^{2} \mathbf{A}=-\mu_{0} \mathbf{J}_{s}
$$

In Equation 2, the wave-number $\kappa=\sqrt{\mu_{0} \epsilon \omega^{2}-i \mu_{0} \sigma \omega}$, where: $i=\sqrt{-1}, \mu_{0}=4 \pi 10^{-7}$ (henry/m) is the magnetic permeability of the free-space, $\epsilon$ is the dielectric permittivity, and $\sigma$ is the electric conductivity. In free space, $\kappa_{0}=\sqrt{\mu_{0} \epsilon_{0}} \omega$. The term that represents the source is the Fourier transform of the current density waveform, $\mathbf{J}_{s}$. We define an oscillating electric dipole at $\left(x_{0}, y_{0}, z_{0}\right)$, as represented in Figure 1, by the following expression:

$$
\mathbf{J}_{s}=I(\omega) d x_{0} \delta\left(x-x_{0}\right) \delta\left(y-y_{0}\right) \delta\left(z-z_{0}\right) \mathbf{i} .
$$

In Equation 3, $I(\omega)$ represents the Fourier transform of the electric current; $d x_{0}$ is the element of length of the $x$-oriented electric dipole, and each $\delta\left(\phi-\phi_{0}\right)$ represents the Dirac delta function with singularity on $\phi=\phi_{0}$. Equation 2 shows that the primary potential, $A_{p}(x, y, z, \omega)$, presents only an $x$-component, and it is given by:

$$
\mathbf{A}_{p}(R, \omega)=\frac{\mu_{0} I(\omega) d x_{0}}{4 \pi} \frac{e^{-i \kappa R}}{R} \mathbf{i}
$$

where: $R=\sqrt{\left(x-x_{0}\right)^{2}+\left(y-y_{0}\right)^{2}+\left(z-z_{0}\right)^{2}}$ is the source-receiver distance. Because $R$ is always positive, we guarantee the convergence of the potential at infinity, assuming that $\Im(\kappa)<0$. Applying Equation 1 in Equation 4 yields the expressions for the primary magnetic field in the frequency domain. 
We obtain the solution for an arbitrary function of the current waveform by convolving this function with the impulse response solution. So, we solve the problem expressing the source current waveform as: $\mathcal{I}(t)=C \delta(t)$ ampere and $I(\omega)=C$ coulomb. We also assume the quasi-static condition, valid for frequencies below $1 \mathrm{MHz}$, and a non-magnetic medium. So $\sigma>>\epsilon \omega$, and we may write either $\kappa \approx$ $\frac{\sqrt{2}}{2} \sqrt{\mu_{0} \sigma \omega}(1-i)$ or $\kappa \approx \sqrt{-\mu_{0} \sigma s}, s=i \omega$. Next, we employ Laplace Transform in Equation 4 to obtain the primary potential and the magnetic field in the time domain due to a dipole in a conductive infinite medium.

$$
\begin{gathered}
\mathbf{a}_{p}(R, t)=\mathcal{F}(R, t) \mathbf{i}, \\
\mathbf{b}_{p}(R, t)=\frac{\mu_{0} \sigma}{2 t} \mathcal{F}(R, t)\left(0 \mathbf{i},-\left(z-z_{0}\right) \mathbf{j},\left(y-y_{0}\right) \mathbf{k}\right), \\
\mathcal{F}(R, t)=\frac{\sqrt{\mu_{0}^{3} \sigma} C d x_{0}}{8 \sqrt{\pi^{3}}} \frac{e^{-\frac{\mu_{0} \sigma R^{2}}{4 t}}}{\sqrt{t^{3}}} u(t),
\end{gathered}
$$

where $u(t)$ is the Heaviside step function, $u(t)=0$ for $t<0$ and $u(t)=1$ for $t>0$.

\section{Dipole in a Three-LAYEREd Medium}

Figure 2 illustrates the geometry of the model. In the air, $z<0, \mu_{0}, \epsilon_{0} \approx 10^{-9} /(36 \pi)($ farad $/ \mathrm{m})$, and $\sigma_{0}=0$. In the sea, $0<z<h_{1}, \mu_{1}=\mu_{0}, \epsilon_{1}=81 \epsilon_{0}$, and $1(\mathrm{~S} / \mathrm{m})<\sigma_{1}<6(\mathrm{~S} / \mathrm{m})$. In the substratum, $z>h_{1}, \mu_{2}=\mu_{0}, \epsilon_{0}<\epsilon_{2}<25 \epsilon_{0}$, and $10^{-3}(\mathrm{~S} / \mathrm{m})<\sigma_{2}<10^{-1}(\mathrm{~S} / \mathrm{m})$.

$$
\begin{aligned}
& z=0 \stackrel{\mu_{0}, \epsilon_{0}, \sigma_{0}=0}{-\mathrm{L} / 2 \longrightarrow \mathrm{L} / 2} \mathrm{X} \\
& z=h_{1} \frac{\mu_{0}, \epsilon_{1}, \sigma_{1}}{\mu_{0}, \epsilon_{2}, \sigma_{2}} \bigsqcup_{\text {学 }} \bullet(x, y, z)
\end{aligned}
$$

Fig. 2 - Illustration of the geometry of the problem with a magnetic tail positioned inside a salty water layer, centered at $y_{0}=0,0 \leq z_{0} \leq h_{1}$ and laid between $-L / 2 \leq x_{0} \leq+L / 2$. Observation point at $(x, y, z)$ of a homogeneous and isotropic three-layered medium.

The secondary potential obeys the homogeneous wave equation and has both the $x$ and the $z$ component for the geometry illustrated in Figure 2. Therefore, for $\eta=x, z$ and $j=0,1,2$ its general solution can be expressed by the following series of Hankels' integrals:

$$
A_{\eta, j}^{S}(x, y, z, \omega)=\sum_{n=0}^{\infty} \cos (n \phi) \int_{0}^{\infty} F_{\eta, j}^{ \pm} e^{ \pm \alpha_{j}\left|z-z_{0}\right|} J_{n}(\lambda r) d \lambda d x_{0},
$$

where: $\cos (\phi)=\frac{\left(x-x_{0}\right)}{r}$ and $\alpha_{j}=\sqrt{\lambda^{2}-\kappa_{j}^{2}}$ is complex with a positive real part. Employing Hankel transform (Sommerfeld 1949), Equation 4 assumes the following expression for the dipole in the water layer:

$$
A_{p, 1}(x, y, z, \omega)=\frac{\mu_{0} I(\omega)}{4 \pi} \int_{0}^{\infty} \frac{\lambda}{\alpha_{1}} e^{-\alpha_{1}\left|z-z_{0}\right|} J_{0}(\lambda r) d \lambda d x_{0}, \quad 0 \leq z_{0} \leq h_{1}
$$


In Equations 7 and $8, J_{n}(\lambda r)$ represents the Bessel Function of first kind and $n$th order and

$$
r=\sqrt{\left(x-x_{0}\right)^{2}+\left(y-y_{0}\right)^{2}} .
$$

Adding the secondary and the primary potentials expressed by Equations 7 and 8, respectively, yields the total potential in the three-layered medium. The solution for the eight functions $F_{\eta, j}^{ \pm}(\lambda)$ is determined by applying the condition of convergence of the potentials at infinity, Maxwell's equations, Equation 1, and the following boundary conditions:

$$
\begin{array}{lll}
B_{z, 0}=B_{z, 1} ; & \mu_{1} B_{x, 0}=\mu_{0} B_{x, 1} ; & \text { on } z=0, \\
E_{y, 0}=E_{y, 1} ; & \mu_{1} B_{y, 0}=\mu_{0} B_{y, 1} ; & \text { on } z=0, \\
B_{z, 1}=B_{z, 2} ; & \mu_{2} B_{x, 1}=\mu_{1} B_{x, 2} ; & \text { on } z=h_{1}, \\
E_{y, 1}=E_{y, 2} ; & \mu_{2} B_{y, 1}=\mu_{1} B_{y, 1} ; & \text { on } z=h_{1} .
\end{array}
$$

\section{The TAIL IN Three-LAyered Media}

We represented the tail by a horizontal line of electric dipoles of length $L$. Figure 2 illustrates both the geometry of the model and the magnetic tail in the sea. By applying the boundary conditions expressed in the system of Equations 9 and integrating along the cable length $\left(x_{0}\right)$, one writes the final expression for $B_{z, 1}(R, \omega)$ due to a magnetic tail situated between the surface and the sea floor.

$$
\begin{aligned}
& B_{z, 1}(R, \omega)=-\frac{\mu_{0}}{4 \pi} I(\omega) \frac{\partial}{\partial y}\left\{\int _ { 0 } ^ { \infty } \left\{\frac{\lambda}{\alpha_{1}} e^{-\alpha_{1}\left|z-z_{0}\right|}+F_{x, 1}^{+}(\lambda) e^{+\alpha_{1} z}\right.\right. \\
&\left.\left.+F_{x, 1}^{-}(\lambda) e^{-\alpha_{1} z}\right\}\left[\int_{-\frac{L}{2}}^{+\frac{L}{2}} J_{0}(\lambda r) d x_{0}\right] d \lambda\right\} \\
& F_{x, 1}^{+}= \frac{\lambda}{\alpha_{1}} \frac{R_{1,2} e^{-2 \alpha_{1} h_{1}}\left(R_{1,0} e^{-\alpha_{1} z_{0}}+e^{+\alpha_{1} z_{0}}\right)}{1-R_{1,0} R_{1,2} e^{-2 \alpha_{1} h_{1}}}, \\
& F_{x, 1}^{-}= \frac{\lambda}{\alpha_{1}} \frac{R_{1,0}\left(R_{1,2} e^{+\alpha_{1} z_{0}} e^{-2 \alpha_{1} h_{1}}+e^{-\alpha_{1} z_{0}}\right)}{1-R_{1,0} R_{1,2} e^{-2 \alpha_{1} h_{1}}} \\
& R_{1, j}=\frac{\alpha_{1}-\alpha_{j}}{\alpha_{1}+\alpha_{j}}, j=0,2 .
\end{aligned}
$$

\section{The magnetic field in the time domain}

To obtain $b_{z, 1}(R, t)$, we apply Fourier or Laplace transform to Equation 10. Only in special cases $b_{z, 1}(R, t)$ is obtained analytically, because $\alpha_{j}, j=1,2,3$ is also a function of $\omega$. In general, it is necessary to perform three numerical integrations: in $\lambda, x_{0}$, and $\omega$. Therefore, the computation of the time domain representation is complex, quite cumbersome, and time consuming as a rule. Let $b_{z, 1}(R, t)=b_{z p}(R, t)+$ 
$b_{z s}(R, t)$. So, we will have for the impulse response that

$$
\begin{aligned}
b_{z p}(R, t)= & \frac{\sqrt{\mu_{0}^{5} \sigma_{1}^{3}} C}{16 \sqrt{\pi^{3}}} \frac{y}{\sqrt{t^{5}}} u(t) \int_{-\frac{L}{2}}^{\frac{+L}{2}} e^{-\frac{\mu_{0} \sigma_{1} R^{2}}{4 t}} d x_{0}, \\
b_{z s}(R, t)= & -\frac{\mu_{0} C}{8 \pi^{2}}\left\{\int _ { - \infty } ^ { + \infty } e ^ { + i \omega t } \left\{\int _ { 0 } ^ { + \infty } \lambda \left\{\frac{F_{x, 1}^{+}(\lambda, \omega)}{\lambda} e^{+\alpha_{1} z}\right.\right.\right. \\
& \left.\left.+\frac{F_{x, 1}^{-}(\lambda, \omega)}{\lambda} e^{-\alpha_{1} z}\right\}\left[\int_{-\frac{L}{2}}^{+\frac{L}{2}} \frac{\partial J_{0}(\lambda r)}{\partial y} d x_{0}\right] d \lambda\right\} d \omega ;
\end{aligned}
$$

and for the step response, $\mathcal{I}(t)=C u(t), I(s)=\frac{C}{s}$, that

$$
\begin{aligned}
b_{z p}(R, t)= & \frac{\mu_{0} C y}{4 \pi} u(t) \int_{-\frac{L}{2}}^{+\frac{L}{2}} \frac{1}{R^{3}}\left\{\operatorname{erfc}\left(\frac{\sqrt{\mu_{0} \sigma_{1}} R}{2 \sqrt{t}}\right)+\frac{\sqrt{\mu_{0} \sigma_{1}} R}{\sqrt{\pi t}} e^{-\frac{\mu_{0} \sigma_{1} R^{2}}{4 t}}\right\} d x_{0}, \\
b_{z s}(R, t)= & -\frac{\mu_{0} C}{8 \pi^{2} i}\left\{\int _ { \beta - i \infty } ^ { \beta + i \infty } e ^ { + s t } \left\{\int _ { 0 } ^ { + \infty } \left\{+\frac{F_{x, 1}^{+}(\lambda, s)}{s} e^{+\alpha_{1} z}\right.\right.\right. \\
& \left.\left.+\frac{F_{x, 1}^{-}(\lambda, s)}{s} e^{-\alpha_{1} z}\right\}\left[\int_{-\frac{L}{2}}^{+\frac{L}{2}} \frac{\partial J_{0}(\lambda r)}{\partial y} d x_{0}\right] d \lambda\right\} d s .
\end{aligned}
$$

Notice that, by taking the time derivative of the expression of the secondary field and substituting $s=i \omega$ in Equation 12, we obtain the equivalent expression of Equation 11.

\section{METHOD OF COMPUTATION}

Presently, there are several available techniques that improve the computation of the field components. We developed one that is particularly suitable for an approximate computation of $b_{z s}(R, t)$ in a highly conductive environment such as the sea water. Next we will describe the procedure. The related algebra is given in the Appendixes A, B, and C.

Write the Fourier transform of the impulse as a time derivative of the Laplace transform of the step. Expand the kernels by the binomial theorem neglecting second and higher order terms of the reflection coefficients. Evaluate the inverse transform of each term of the expansion in $s=i \omega$ by a deformation of the Bromwich path $(\mathrm{Br})$ into a closed contour. Evaluate the Hankel $(\lambda)$ and the tail $\left(x_{0}\right)$ integrals in the best order. By employing Equation A1 of Appendix A and Equations B1, B2, and B4 of Appendix B, we rewrite Equation 11 and express $b_{z, 1}(R, t)$ as:

$$
\begin{aligned}
& b_{z p}(R, t)=\frac{\pi y \sigma_{1}}{10^{5} t^{2}} e^{-\left(\left(\frac{y}{2 \chi}\right)^{2}+\left(\frac{z-z_{0}}{2 \chi}\right)^{2}\right)}\left\{\operatorname{erf}\left(\frac{x+\frac{L}{2}}{2 \chi}\right)-\operatorname{erf}\left(\frac{x-\frac{L}{2}}{2 \chi}\right)\right\} u(t) \\
& b_{z s}(R, t)=100 y \int_{0}^{+\infty} \dot{f}_{x, 1}(\lambda, t)\left[\int_{-\frac{L}{2}}^{+\frac{L}{2}} \frac{J_{1}(\lambda r)}{r} d x_{0}\right] \lambda d \lambda .
\end{aligned}
$$

In Equation 13 we made $C=1$ coulomb, substituted the value of $\mu_{0}$ in henry/m, employed time in second, and multiplied by $10^{9}$ to obtain $b_{z, 1}(R, t)$ in nanotesla, with the unit of $\dot{f}_{x, 1}(\lambda, t)$ in hertz. Adding 
the partial derivative with respect to time of the terms of Equation B1, of Equations B2 and B3, and of Equations B4, B5, and B6 of Appendix B, we express $\dot{f}_{x, 1}(\lambda, t)$ approximately as:

$$
\begin{aligned}
\dot{f}_{x, 1}(\lambda, t) \approx & \left\{\frac{\lambda e^{-\lambda^{2} \chi^{2}}}{\mu_{0} \sigma_{1} \chi \sqrt{\pi}} \sum_{j=1}^{4}(-1)^{j} e^{-\frac{q_{j}^{2}}{4 \chi^{2}}}+\sum_{j=1}^{4, j \neq 3}(-1)^{j+1} \frac{\partial g_{j}^{+}}{\partial t}\right. \\
& \left.+\sum_{j=2}^{4}(-1)^{j+1}\left(\frac{\partial g_{j}^{-}}{\partial t}+\frac{\partial h_{j}^{-}}{\partial t}\right)\right\} u(t) .
\end{aligned}
$$

Substituting Equation 14 in Equation 13 with the help of Equations B1, B2, B4, B3, B5, and B6, we obtain the following expression for $b_{z s}(R, t)$ :

$$
\begin{aligned}
& b_{z s}(R, t)=100 y \int_{-\frac{L}{2}}^{+\frac{L}{2}}\left\{\int _ { 0 } ^ { + \infty } 2 \lambda \left\{\sum_{j=1}^{4}(-1)^{j} e^{-\frac{q_{j}^{2}}{4 \chi^{2}}} \frac{e^{-\lambda^{2} \chi^{2}}}{2 \sqrt{\pi} \mu_{0} \sigma_{1} \chi}\right.\right. \\
& +\sum_{j=1}^{4, j \neq 3}(-1)^{j+1} e^{-\frac{q_{j}^{2}}{4 \chi^{2}}} \frac{e^{-\lambda^{2} \chi^{2}}}{\sqrt{\pi} \mu_{0}\left(\sigma_{1}-\sigma_{2}\right) \chi}+\sum_{j=1}^{4, j \neq 3}(-1)^{j+1} \frac{\lambda}{\mu_{0}\left(\sigma_{1}-\sigma_{2}\right)} \\
& \times\left\{\frac{e^{-\lambda q_{j}}}{2} \operatorname{erfc}\left(-\lambda \chi+\frac{q_{j}}{2 \chi}\right)-\frac{e^{+\lambda q_{j}}}{2} \operatorname{erfc}\left(\lambda \chi+\frac{q_{j}}{2 \chi}\right)\right\} \\
& +\sum_{j=1}^{4, j \neq 3}(-1)^{j+1} \frac{\sqrt{\sigma_{2}} \sqrt{p_{1}}}{\pi \sqrt{\mu_{0}}} \int_{0}^{1} \frac{\sqrt{1-p} \sin \left(\xi_{j} \sqrt{p_{1}}\right)}{\left(\sigma_{1}-\sigma_{2}\right) p+\sigma_{2}} e^{-\left(\gamma+p_{1} p\right) t} d p \\
& +\sum_{j=2}^{4}(-1)^{j+1} \frac{e^{-\gamma t}}{\sqrt{\mu_{0} \sigma_{1}}}\left\{\frac { \lambda \chi e ^ { + \lambda ^ { 2 } \chi ^ { 2 } } } { 2 \sqrt { t } } \left\{e^{-\lambda q_{j}} \operatorname{erfc}\left(-\lambda \chi+\frac{q_{j}}{2 \chi}\right)\right.\right. \\
& \left.-e^{+\lambda q_{j}} \operatorname{erfc}\left(\lambda \chi+\frac{q_{j}}{2 \chi}\right)\right\}-\frac{\sqrt{\gamma+\frac{\sigma_{1}}{\epsilon_{0}}} e^{+\gamma t+\frac{\sigma_{1}}{\epsilon_{0}} t}}{2} \\
& \times\left\{e^{-\sqrt{\gamma+\frac{\sigma_{1}}{\epsilon_{0}}} \sqrt{\mu_{0} \sigma_{1}} q_{j}} \operatorname{erfc}\left(-\sqrt{\left(\gamma+\frac{\sigma_{1}}{\epsilon_{0}}\right)} t+\frac{q_{j}}{2 \chi}\right)\right. \\
& \left.\left.-e^{+\sqrt{\gamma+\frac{\sigma_{1}}{\epsilon_{0}}} \sqrt{\mu_{0} \sigma_{1}} q_{j}} \operatorname{erfc}\left(+\sqrt{\left(\gamma+\frac{\sigma_{1}}{\epsilon_{0}}\right) t}+\frac{q_{j}}{2 \chi}\right)\right\}\right\} \\
& +\sum_{j=2}^{4}(-1)^{j+1} \frac{c_{0}}{\pi} \int_{0}^{+\infty} \frac{\sqrt{\left(p+p_{0}\right)\left(p+\dot{p}_{0}\right)} \sin \left(\xi_{j}\right) e^{-(\gamma+p) t}}{(p+\gamma)\left(p+\gamma+\frac{\sigma_{1}}{\epsilon_{0}}\right)} d p \\
& -\sum_{j=2}^{4}(-1)^{j+1} \frac{2 c_{0}}{\pi} \int_{0}^{c_{0} \lambda}\left\{\frac{\sigma_{1}}{\epsilon_{0}} \frac{\sin \left(p t-b_{j}\right)}{p}+\cos \left(p t-b_{j}\right)\right\} \\
& \left.\left.\times \frac{\sqrt{c_{0}^{2} \lambda^{2}-p^{2}} e^{-a_{j}}}{\frac{\sigma_{1}^{2}}{\epsilon_{0}^{2}}+p^{2}} d p\right\} J_{1}(\lambda r) \lambda d \lambda\right\} \frac{d x_{0}}{r}, \\
& \xi_{j}=\sqrt{\mu_{0} \sigma_{1}} q_{j} \sqrt{p}
\end{aligned}
$$


Equation 13 shows that the primary field is independent of the thickness of the liquid layer. On the other hand, the secondary field depends on it and is made up of an infinite sequence of terms, of which we computed only the first four of them as shows Equation 15. Though the electromagnetic energy, in fact, scatters at each interface, we can associate the series to an infinite sequence of reflections at the top and bottom of the liquid layer, by analogy with the ray theory. Therefore, Equation 15 individualizes the wavefield components, and we may employ it for data processing such as filtering and continuation operations, as well as decomposition into upward and downward terms (Amundsen et al. 2006). Furthermore, Equation 15 is already equivalent to a multiple suppression of the exact equation.

Recall that both $a_{j}$ and $b_{j}$ are linear functions of $q_{j}$. We may associate $q_{j}$ respectively to: a single reflection at the bottom $-q_{1}$; a single reflection at the surface $-q_{3}$; a reflection at the surface followed by a reflections at the bottom $-q_{2}$; and a reflection at the bottom followed by a reflection at the surface $-q_{4}$. The first term of Equation 15 contains all four $q_{j}$. It is the leading term and only depends on the properties of the sea. It would be the only one if the air and the substratum were perfect conductors. It is of the same order of magnitude and of opposite sign to the primary field. The following three terms contain the contribution of the conductive substratum, primarily via $q_{1}$, and secondarily via $q_{2}$ and $q_{4}$. They don't have the term in $q_{3}$, and the first one is similar to the main term. The last three terms contain the contribution from the air-sea interface via $q_{3}, q_{2}$, and $q_{4}$, and they don't have the term in $q_{1}$. In Appendix C we develop the first two terms on the right-hand side of Equation 15 to obtain Equations $\mathrm{C} 4$ and $\mathrm{C} 5$. We computed the other five terms of Equation 15 numerically.

\section{Alternative representations for the reflection coefficient}

If the conductivity contrast between two sea water layers or between the sea water and its substratum is sufficiently small: $\sigma_{1}-\sigma_{2}<<1$, we may express the reflection coefficient approximately as:

$$
R_{1,2} \approx \frac{\left(1-\frac{\sigma_{2}}{\sigma_{1}}\right) s}{4\left(s+\frac{\lambda^{2}}{\mu_{0} \sigma_{1}}\right)}
$$

If, however, the contrast is very large, we may expand $R_{1,2}$ approximately as:

$$
R_{1,2}=\left(1-\frac{\alpha_{2}}{\alpha_{1}}\right) \sum_{n=0}^{+\infty}(-1)^{n}\left(\frac{\alpha_{2}}{\alpha_{1}}\right)^{n} \approx 1-2 \frac{\alpha_{2}}{\alpha_{1}}
$$

In this second case, a procedure similar to the one developed in Appendix B gives the following alternative representation for $\dot{f}_{x, 1}(\lambda, t)$ of Equation 13:

$$
\dot{f}_{x, 1}(\lambda, t) \approx-\frac{\lambda^{2} e^{-\lambda^{2} \chi^{2}}}{\mu_{0} \sigma_{1}}\left\{4-\frac{\sigma_{2}}{\sigma_{1}}-\frac{e^{-\left(\frac{z-z_{0}}{2 \chi}\right)^{2}}+e^{-\left(\frac{z+z_{0}}{2 \chi}\right)^{2}}}{\lambda \chi \sqrt{\pi}}\right\} .
$$

To compute the secondary field, we perform the Hankel transform from the $\lambda$ domain in Appendix D before integrating in $x_{0}$. The transform of the third term of Equation 16 yields the Error Function. The transform of the first two terms yields either a partial $y$ derivative of Kummer's confluent hypergeometric 
series or a generalized Laguerre polynomial of order 1/2 and coefficient 1 (Erdélyi 1954, Abramowitz and Stegun 1968). We determined this particular Laguerre polynomial employing fractional derivative (Sokolov et al. 2002) and obtained Equation D4.

\section{RESULTS OF THE NUMERICAL SIMULATIONS}

The following parameters are constants in the maps and graphs: $\sigma_{1}=3 \mathrm{~S} / \mathrm{m}$, which is an average value for the conductivity of sea water; $L=300 \mathrm{~m} ; x_{0}=y_{0}=0$; and $z=h_{1}^{-}$. We also set $t=0.001 \mathrm{~s}$ in the maps, and $x=0 \mathrm{~m}$ and $y=20 \mathrm{~m}$ in the graphs. The caption of each figure contains the values of the other parameters.

We set the sea layer thickness, $h_{1}$, equal to $20 \mathrm{~m}$ and $100 \mathrm{~m}$ to simulate, respectively, a shallow water and an intermediate depth environment. For surveys at the sea surface, as in magnetic submarine countersweep campaigns, $z_{0}=z_{0}^{(1)}=0$. For ocean bottom geophysical surveys, $z_{0}=z_{0}^{(2)}=z=h_{1}^{-}$. We employed three values for the conductivity of the substratum, $\sigma_{2}$, to cover the average range of variation of the conductitivity of rocks: $0.3 \mathrm{~S} / \mathrm{m} ; 0.03 \mathrm{~S} / \mathrm{m}$, and $0.003 \dot{\mathrm{S}} / \mathrm{m}$.

In the case of the maps, it is sufficient to represent only one quadrant of them, because all the variations are even with respect to $x$ and odd with respect to $y$. We computed the functions described by Equations 13,14 , and 15 for values of time up to $10^{-2} \mathrm{~s}$ because they decay steadly for $t>10^{-2} \mathrm{~s}$.

The graphs of Figure 3 represent the primary and the total field at the bottom of a $20 \mathrm{~m}$ water layer, overlying a substratum in which $\sigma_{2}=0.3 \mathrm{~S} / \mathrm{m}$. Both the magnitude and the time displacement of the peaks of the curves are compatible with the source-receiver distances. The graphs of Figure 4 represent the primary and the total field at the bottom of an $100 \mathrm{~m}$ water layer, also overlying a substratum in which $\sigma_{2}=0.3 \mathrm{~S} / \mathrm{m}$. In this case, the magnitude and the time displacement of the peaks of the curves are compatible only for the primary field. The magnitude of the total field in Figure 4(c) is larger than in Figure 4(d), in spite of a smaller receiver-transmitter distance in this last one. The magnitude of the total field differs substantially between Figures 3(d) and 4(d), even though they have the same transmitterreceiver configuration. These two facts show the role that the thickness of the sea water layer plays in the secondary field.

If we neglect the length of the tail and assume the origin of the pulse at its center, we can estimate a group velocity, $V_{g}$, of the peak for the primary field under the conditions of Figures 3 and 4 . The precision will be better for a larger transmitter-receiver distance. For instance: for Figure $4(\mathrm{a}), V_{g} \approx 25 \mathrm{~km} / \mathrm{s}$ : and for Figure 3(a), $V_{g} \approx 70 \mathrm{~km} / \mathrm{s}$. In the present case, the phase velocity for the plane wave approximation under quasi-static conditions is given by $V_{f}=10^{3} \sqrt{3.3 f}$. Since we may express the group velocity as:

$$
V_{g}=\frac{V_{f}}{1-\kappa \frac{d V_{f}}{d \omega}},
$$

we may conclude that $V_{g} \approx 2 V_{f} \approx 10^{3} \sqrt{13.3 f}$. So, the energy of the signal is centered, respectively, about $47 \mathrm{~Hz}$ and $370 \mathrm{~Hz}$ on Figures 4(a) and 3(a). This fact and the definition of the quality factor deserves further investigation, because it is useful to select the on and off times of a square or a pseudosquare source waveform. 


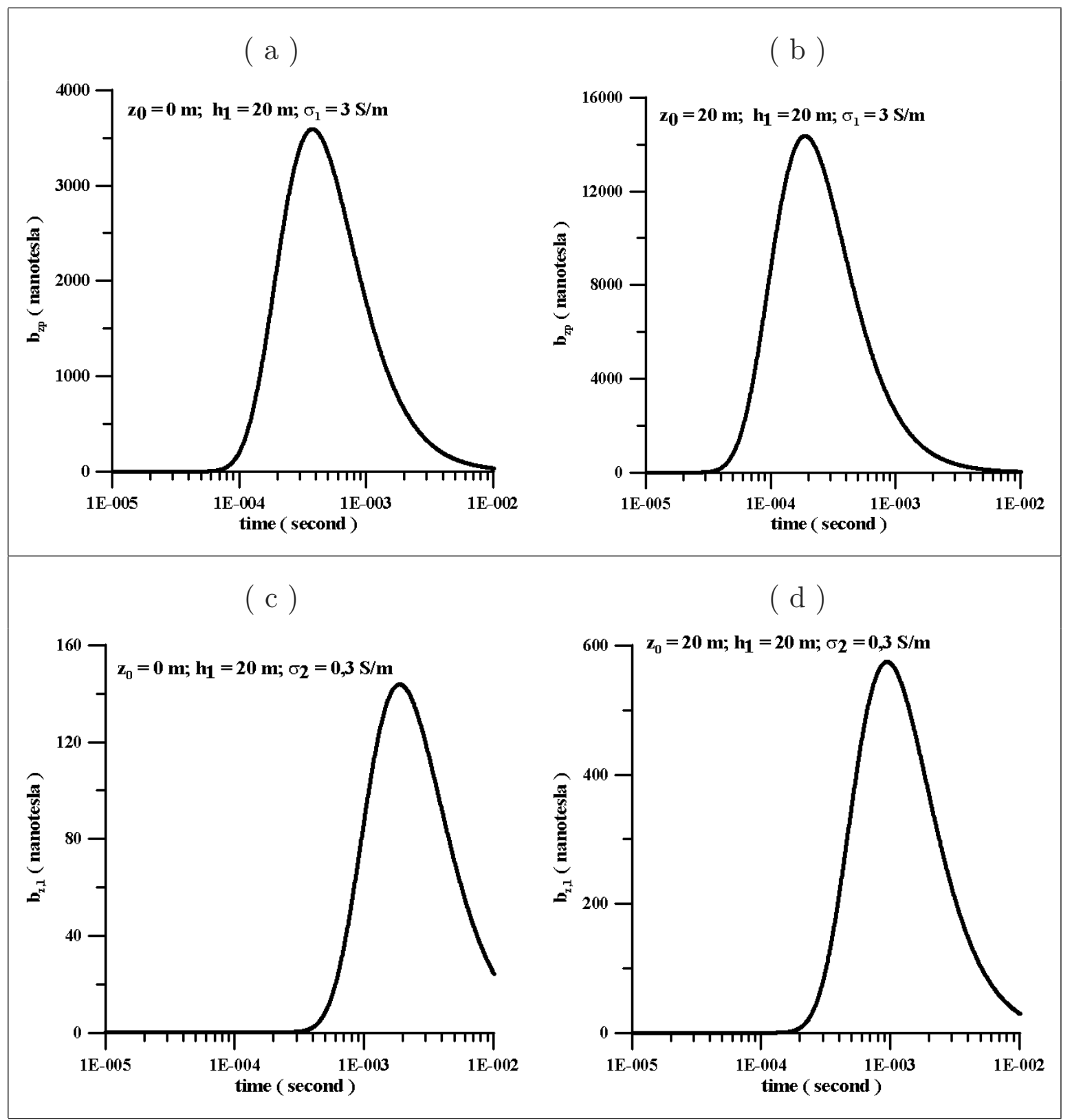

Fig. 3-Graphs of the time variation of the vertical component of the magnetic field for the case of a water layer with $h_{1}=20 \mathrm{~m}$ and $\sigma_{1}=3 \mathrm{~S} / \mathrm{m}$, overlying a basement with $\sigma_{2}=0.3 \mathrm{~S} / \mathrm{m}$. Primary: $b_{z p}$. Total: $b_{z, 1}$.

The contour maps of Figure 5 display the variation of the primary and the total field at the bottom of a $20 \mathrm{~m}$ water layer, overlying a substratum in which $\sigma_{2}=0.3 \mathrm{~S} / \mathrm{m}$. All the maps show a perfect delineation of the tail position, and their magnitudes are also compatible with the transmitter-receiver distances. The contour maps of Figure 6 display the variation of the primary and the total field at the bottom of a $100 \mathrm{~m}$ water layer, also overlying a substratum in which $\sigma_{2}=0.3 \mathrm{~S} / \mathrm{m}$. Only the primary field map shows a perfect delineation of the tail position and a field magnitude compatible with the transmitter-receiver distance. The two maps of the total field reflect the influence of the larger water layer thickness. 


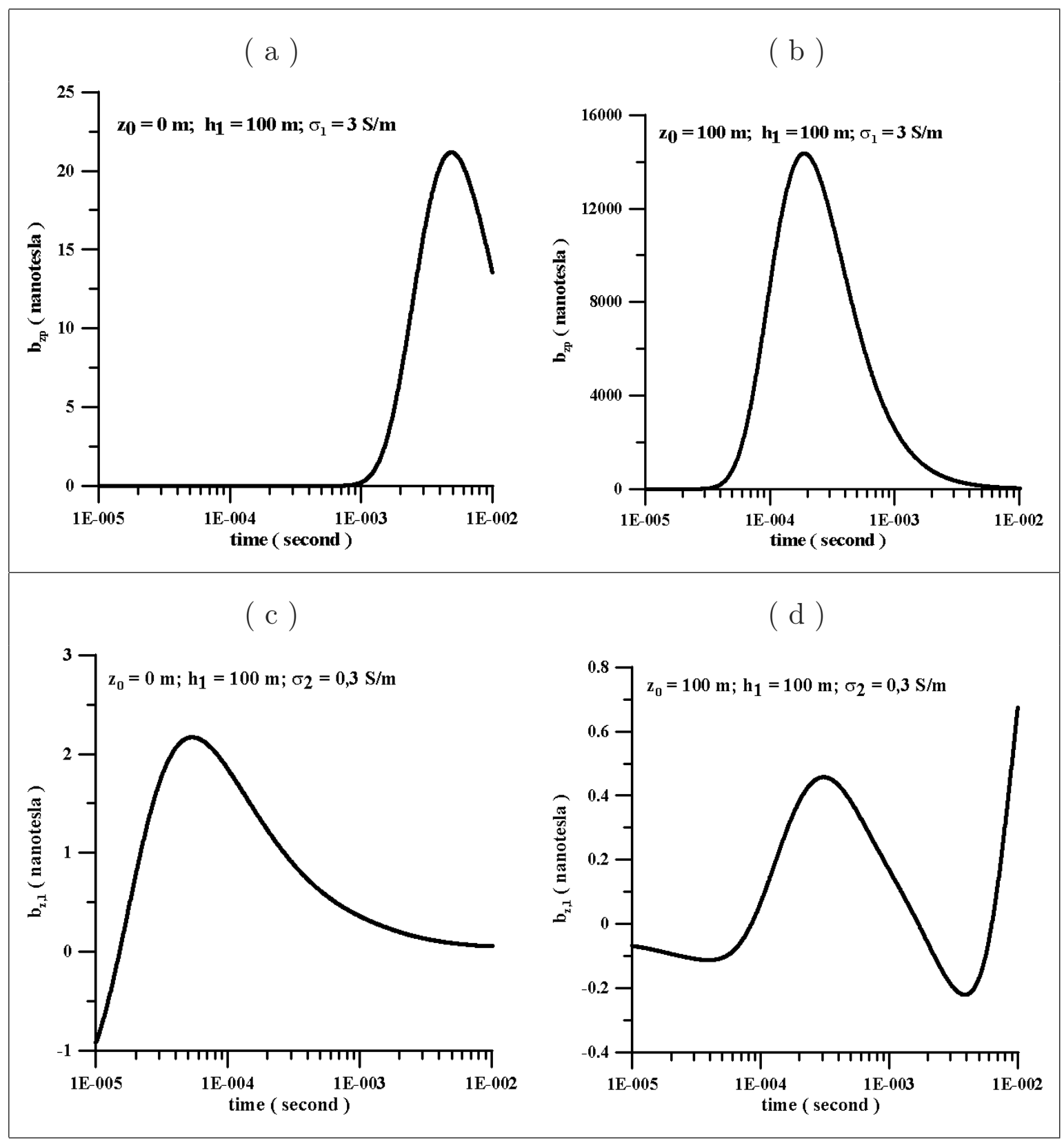

Fig. 4 - Graphs of the time variation of the vertical component of the magnetic field for the case of a water layer with $h_{1}=100 \mathrm{~m}$ and $\sigma_{1}=3 \mathrm{~S} / \mathrm{m}$, overlying a basement with $\sigma_{2}=0.3 \mathrm{~S} / \mathrm{m}$. Primary: $b_{z p}$. Total: $b_{z, 1}$.

The primary field reaches the higher values along a strip on each side of the tail. The longitudinal extension and transversal position of the peak depend on time and on the vertical distance between the tail and the receiver. As an average, the peak covers $80 \%$ of the length of the tail and spreads at a transversal distance of approximately $6 \%$ of the same length. Let us assume for those maps an average displacement of $18 \mathrm{~m}$ at $t=0.001 \mathrm{~s}$. Under the present circumstances, we obtain $V_{g} \approx 18 \mathrm{~km} / \mathrm{s}$. This suggests that the energy of the signal is centered about $24 \mathrm{~Hz}$ at that instant. 


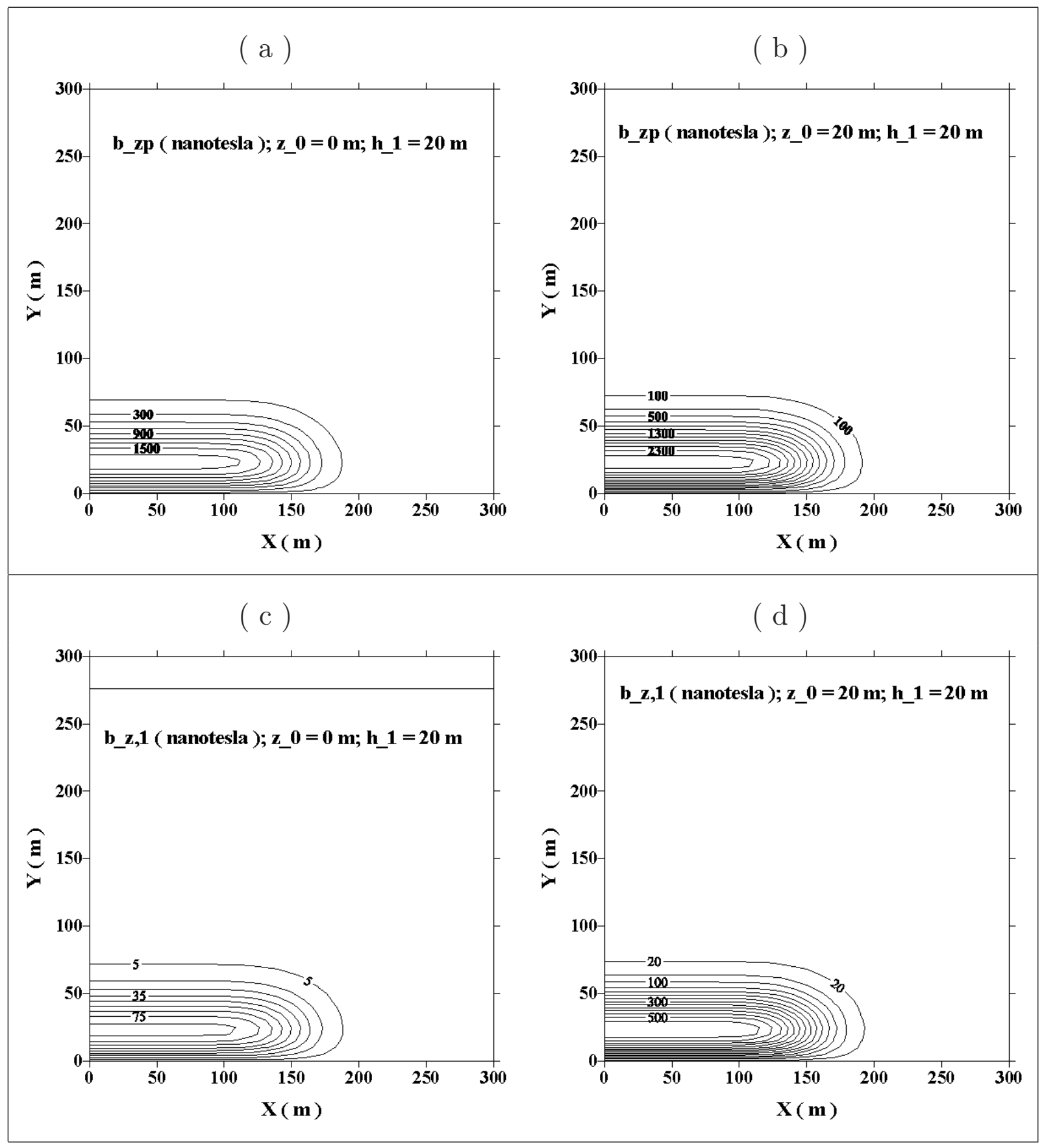

Fig. 5 - Contour maps of the vertical component of the magnetic field for the case of a water layer with $h_{1}=20 \mathrm{~m}$ and $\sigma_{1}=3 \mathrm{~S} / \mathrm{m}$ and a substratum with $\sigma_{2}=0.3 \mathrm{~S} / \mathrm{m}$. Primary: $b_{z p}$. Total: $b_{z, 1}$.

The graphs of Figure 7 show the contribution of the substratum to the secondary field for three values of $\sigma_{2}: 0.3 \mathrm{~S} / \mathrm{m}, 0.03 \mathrm{~S} / \mathrm{m}$, and $0.003 \mathrm{~S} / \mathrm{m}$. Together they show the influence that the conductivity of the substratum, the position of the transmitter and the water layer thickness have on the time variation of the field. The change in sign of the three terms of the series of Equation 15 and the variation of $q_{j}$ help to understand the behavior of the curves. There is a paradox related to the peak values in this figure. 


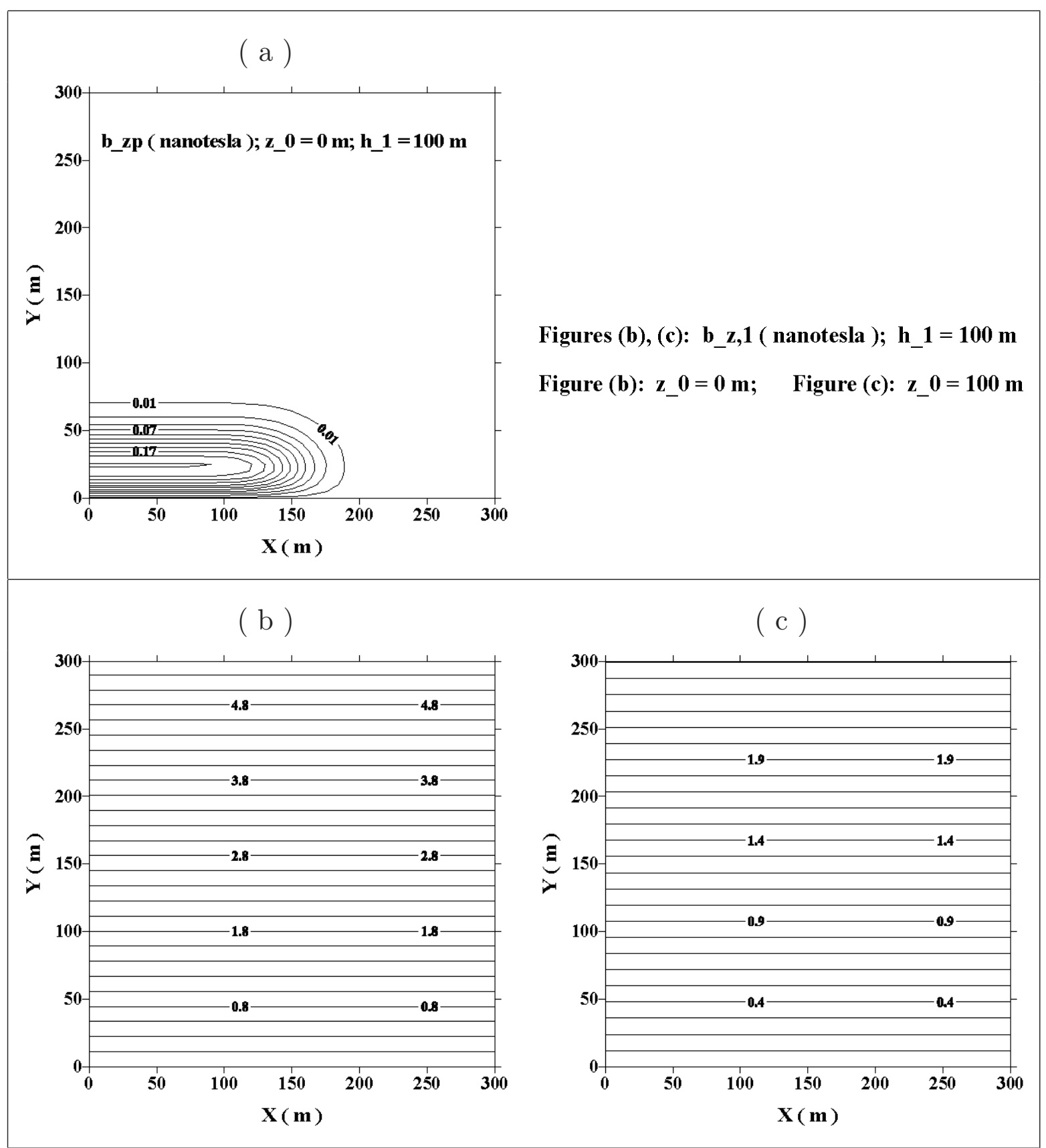

Fig. 6-Contour maps of the vertical component of the magnetic field for the case of a water layer with $h_{1}=100 \mathrm{~m}$ and $\sigma_{1}=3 \mathrm{~S} / \mathrm{m}$ and a substratum with $\sigma_{2}=0.3 \mathrm{~S} / \mathrm{m}$. Primary: $b_{z p}$. Total: $b_{z, 1}$.

Figure 7(b) has a peak value five times larger than the peak value of Figure 7(a) for $\sigma_{2}=0.03 \mathrm{~S} / \mathrm{m}$ and even larger for $\sigma_{2}=0.003 \mathrm{~S} / \mathrm{m}$, but the magnitude ratio is inverse for $\sigma_{2}=0.3 \mathrm{~S} / \mathrm{m}$. A similar behavior occurs between Figures 7(d) and 7(c). However, the curve for $\sigma_{2}=0.03 \mathrm{~S} / \mathrm{m}$ presents a value smaller than the curve for $\sigma_{2}=0.003 \mathrm{~S} / \mathrm{m}$ in graph (d), while the contrary occurs in graph (b). Therefore, the graphs of Figure 7 indicate how to improve the choice of the transmitter distance to the bottom of the sea for each water layer thickness and for each conductivity of the top layer of the sea substratum. 


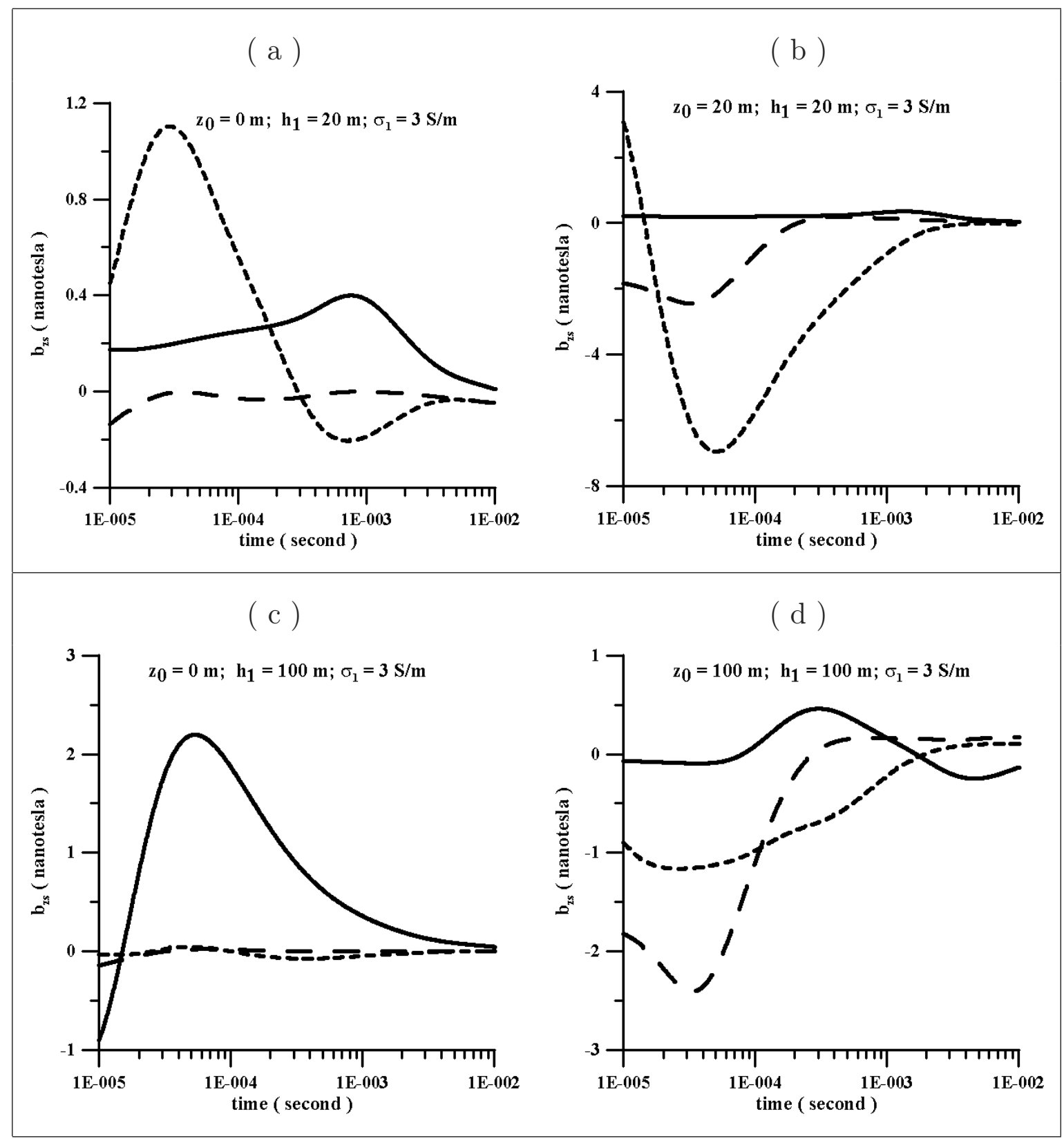

Fig. 7 - Graphs of the time variation of the main contribution of the substratum for the vertical component of the magnetic field: $\sigma_{2}$ represents the conductivity of the substratum. (a) and (b) $h_{1}=20 \mathrm{~m}$. (c) and (d) $h_{1}=100 \mathrm{~m}$. Solid line: $\sigma_{2}=0.3 \mathrm{~S} / \mathrm{m}$. Dotted line: $\sigma_{2}=0.03 \mathrm{~S} / \mathrm{m}$. Dashed line: $\sigma_{2}=0.003 \mathrm{~S} / \mathrm{m}$.

In order to evaluate the discrimination capability of the electromagnetic method in the present situation, we also computed the difference between the values of the field for two different substratuns. The contour maps of Figures 8, 9, and 10 display the results, respectively, for the following pairs of conductivity values: $0.3 \mathrm{~S} / \mathrm{m}$ and $0.03 \mathrm{~s} / \mathrm{m} ; 0.3 \mathrm{~S} / \mathrm{m}$ and $0.003 \mathrm{~S} / \mathrm{m}$; and $0.03 \mathrm{~S} / \mathrm{m}$; and $0.003 \mathrm{~S} / \mathrm{m}$. All the maps display a monotonic behavior with respect to the transverse distance to the tail. Except for the map of Figure 9, under an $100 \mathrm{~m}$ water layer, the values have the same order of magnitude of the total field values: 
see maps of Figure 6(c) and (d). Under a $20 \mathrm{~m}$ water layer, the magnitudes are also of the same order of the total field of the maps of Figure 5 for transverse distances to the tail larger than $75 \mathrm{~m}$. This means that, in shallow waters, it is necessary to separate the receivers at least one quarter of the tail lenght to achieve an adequate discrimination. Though this is not a problem in deep waters, one should consider the possibility of adverse situations as the referred case of Figure 9(d).

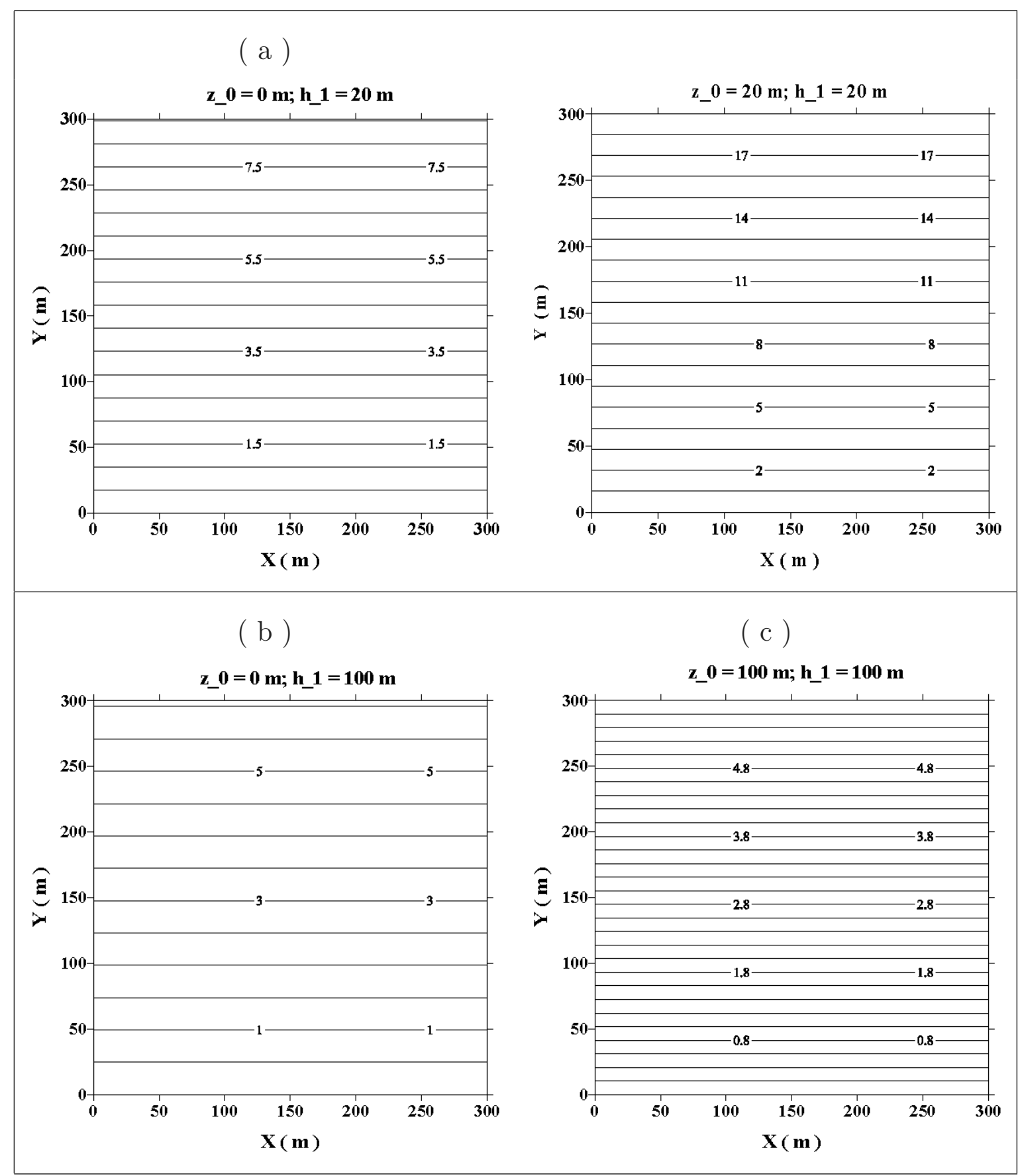

Fig. 8 - Contour maps of the difference of the vertical component of the magnetic field in nanotesla between one substratum with $\sigma_{2}=0.3 \mathrm{~S} / \mathrm{m}$ and another with $\sigma_{2}=0.03 \mathrm{~S} / \mathrm{m}$. $h_{1}$ represents the thickness of the sea layer. 


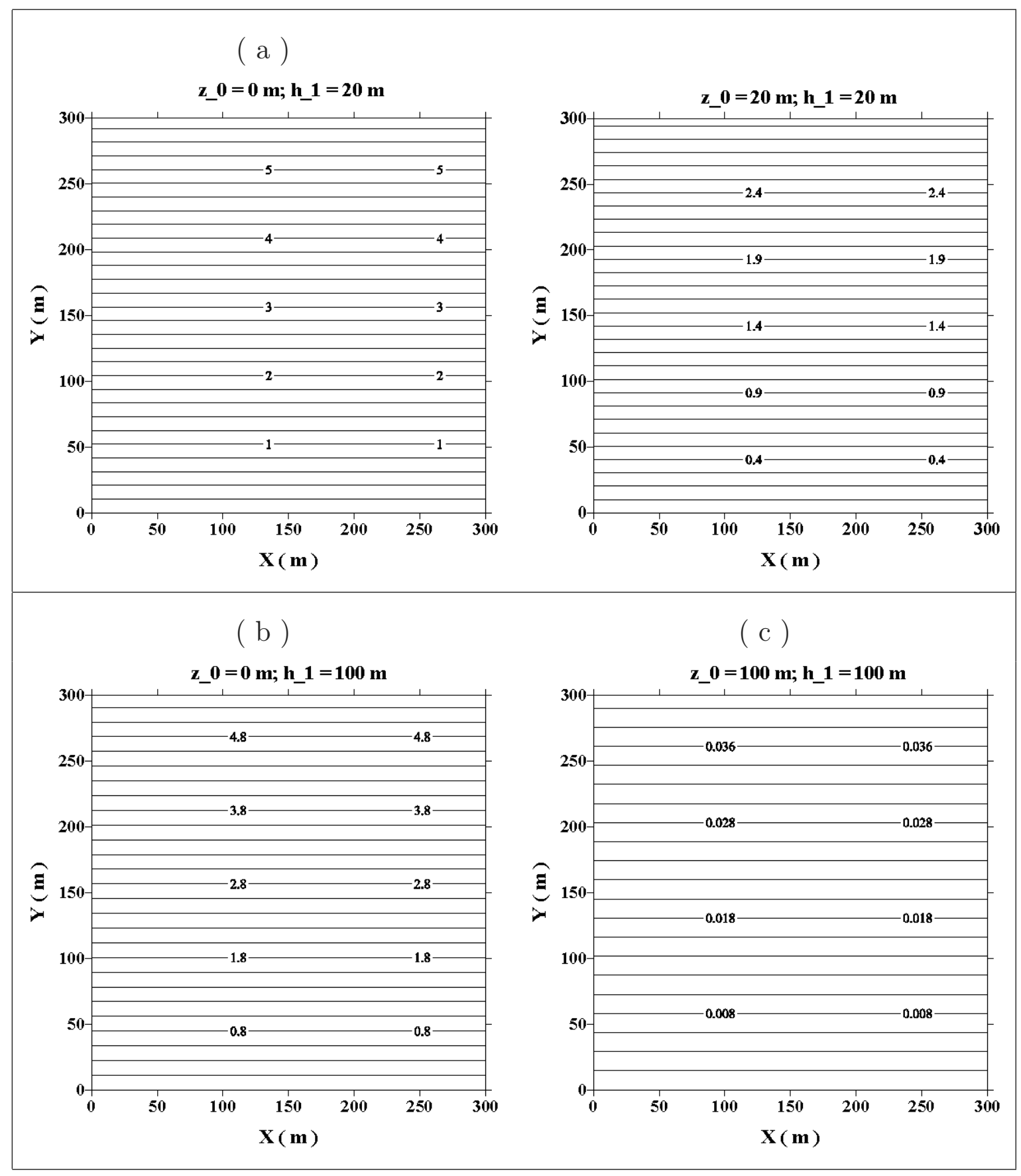

Fig. 9 - Contour maps of the difference of the vertical component of the magnetic field in nanotesla between one substratum with $\sigma_{2}=0.3 \mathrm{~S} / \mathrm{m}$ and another with $\sigma_{2}=0.003 \mathrm{~S} / \mathrm{m}$. $h_{1}$ represents the thickness of the sea layer.

\section{CONCLUSIONS}

We solved the induced electromagnetic field both in the frequency and in the time domain for tail and receiver in the sea. The integrands are complex functions with multiple branch points. So, it is difficult to represent the field as a closed expression or with a minimum number of integrals. We approached the 


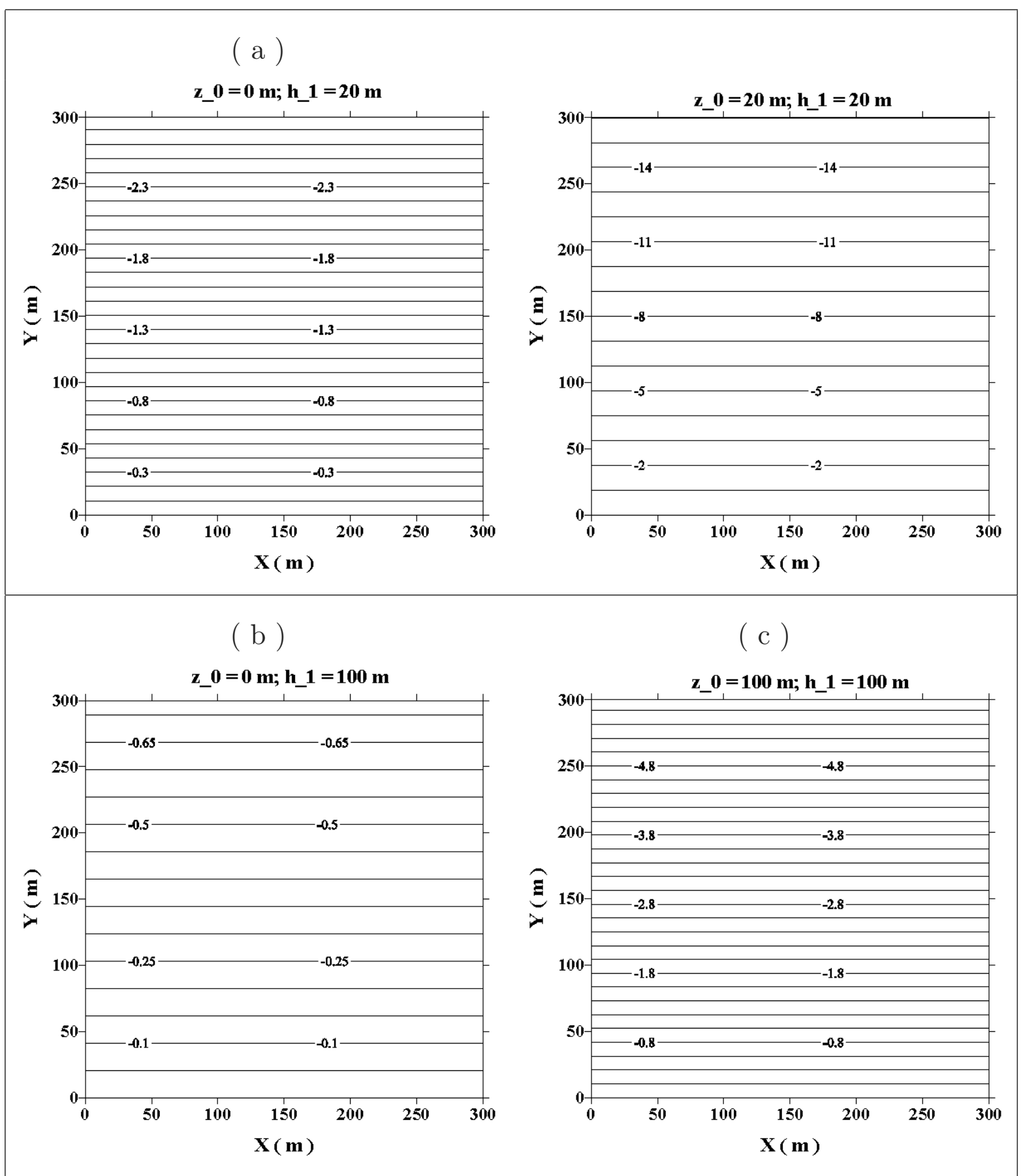

Fig. 10 - Contour maps of the difference of the vertical component of the magnetic field in nanotesla between one substratum with $\sigma_{2}=0.03 \mathrm{~S} / \mathrm{m}$ and another with $\sigma_{2}=0.003 \mathrm{~S} / \mathrm{m}$. $h_{1}$ represents the thickness of the sea layer.

expression for a pulse source of current by a Dirac Delta function. Though it describes an abstract model for the source, its analysis is useful to: evaluate noises caused by current peaks; separate the terms of the wave-field due to single or double reflections and to the electrical parameters; and solve the case of a generic source waveform employing convolution. 
Though the source signal consists of a single pulse whose time duration is negligible, the conductivity of the sea water changes it appreciably. It increases its duration and causes the primary and the secondary fields to be measured up to times later than tens of milliseconds depending on the source-receiver offset. The curves of the graphs show this fact clearly, as well as the effect of the thickness of the sea layer on the relative magnitude of both the primary and the secondary fields. The contour maps show the influence of the thickness of the sea layer on the spatial variation of the field.

The model is adequate for shallow, intermediate, and deep water environments. For safety procedures, a step source model will be useful to evaluate transient fields at turn on and turn off times during field operations. The results provide a wealth of information depending on the selected source-receiver configuration and the conductivity contrasts. The analysis, modelling, and interpretation of these data help to: define safety procedures in counter-sweep campaigns; map the conductivity of the submarine soil in environmental studies of marshes and bays; and identify, as a first approach, deep electrical structures, including possible economical deposits under the sea, employing mCSEMI (marine controlled source electromagnetic induction) methods in exploration and development works.

There are situations as show the maps of Figure 9, in which even for a high conductivity contrast the discrimination capability is poor. However, in general, the analysis of the magnitudes as a function of the electrical conductivity contrast, of the water layer thickness, of the transverse distance to the tail, and of the vertical position of the tail leads to evaluate the discrimination capability in the following way:

- it can be adequate for any conductivity contrast of the substratum as long as the measurement accuracy is better than about $0.01 \mathrm{nT}$;

- in shallow water it improves by increasing the receivers' distance across the axis of the source and decreasing the distance between the tail and the seafloor; and

- for deep water it is in general adequate for any position of the receivers.

\section{ACKNOWLEDGMENTS}

This work has been supported with a grant and a fellowship from Conselho Nacional de Desenvolvimento Científico e Tecnológico (CNPq).

\section{RESUMO}

A análise de campos eletromagnéticos causados por correntes alternadas ou transientes fluindo ao longo de um cabo na água do mar tem várias aplicações. Ela provê suporte à interpretação de dados geofísicos eletromagnéticos e aos procedimentos de segurança contra a ameaça de minas submarinas. A abordagem do problema emprega um potencial vetorial magnético, no domínio da frequência, devido a um dipolo elétrico com uma fonte tipo pulso e calcula transformações de Laplace e de Hankel e integração ao longo do comprimento do cabo, para descrever a variação temporal do campo magnético de indução devido a um dipolo elétrico de comprimento finito. O resultado é aplicável em ambientes de água do mar rasa ou profunda, adaptável para qualquer forma de onda de corrente transmitida e útil para separação de campos de ondas. As configurações se referem a uma bobina receptora horizontal no fundo do mar e simulam: uma campanha de varredura de minas submarinas com uma fonte de corrente na superfície ou um 
levantamento geofísico com uma fonte de corrente próxima ao assoalho do mar. Portanto, a presente análise pode servir: para definir parâmetros em contra-varredura de minas submarinas; para mapear a condutividade de sedimentos sob águas rasas em prevenção e controle de contaminação e como uma primeira abordagem na caracterização de depósitos minerais e de hidrocarbonetos no mar.

Palavras-chave: dipolos, energia eletromagnética, campos induzidos, mar.

\section{REFERENCES}

Abramowitz M and Stegun IA. 1968. Laplace Transforms. In: Abramowitz M and Stegun IA (Eds), Handbook of Mathematical Functions, Washington: National Bureau of Standards, p. 1020-1029.

Amundsen L, Løseth L, Mittet R, Ellingsrud S and Ursin B. 2006. Decomposition of electromagetic fields into upgoing and downgoing components. Geophysics 71: G211-G223.

BATISTA LS AND SAMPAIO EES. 2003. Scattering of electromagnetic plane waves by a buried vertical dike. An Acad Bras Cienc 75: 189-207.

Chave AD, Constable SC and Edwards RN. 1991. Electrical Exploration Methods for the Seafloor. In: Nabighian MN (Ed), Electromagnetic Methods in Applied Geophysics, vol. II, Application, Part B, Chapter 12, Tulsa SEG, p. 931-966.

Chave AD AND Cox CS. 1982. Controlled electromagnetic sources for measuring electrical conductivity beneath the the oceans, 1: forward problem and model study. Jour Geophys Res 87: 5327-5338.

Cheesman SJ, Edwards RN And Chave AD. 1987. On the theory of seafloor conductivity mapping using transient electromagnetic systems. Geophysics 52: 204-217.

Constable S. 2006. Marine electromagnetic methods - a new tool for offshore exploration. The Leading Edge 25: $438-445$.

Constable S And Cox CS. 1996. Marine controlled-source electromagnetic sounding 2. The PEGASUS experiment. Jour Geophys Res 101: 5519-5530.

CONSTABLE S AND WeisS CJ. 2006. Mapping thin resistors and hydrocarbons with marine EM methods: insights from 1D modeling. Geophysics 71: G43-G51.

EDWARDS RN. 1997. On the resource evaluation of marine gas hydrate deposits using sea-floor transient electric dipole-dipole methods. Geophysics 62: 63-74.

Eidesmo T, Ellingsrud S, MacGregor LM, Constable S, Sinha MC, Johansen S, Kong FN and Westerdah H. 2002. Sea bed logging (SBL), a new method for remote and direct identification of hydrocarbon filled layers in deepwater areas. First Break 20: 144-152.

Ellingsrud S, Eidesmo T, Johansen S, Sinha MC, MacGregor LM and Constable S. 2002. Remote sensing of hydrocarbon layers by seabed logging (SBL): results from a cruise offshore Angola. The Leading Edge 21: 972-982.

ERdÉLYi A (Ed). 1954. Tables of integral transforms, v. I, McGraw-Hill Book Co., New York.

FlosadótTiR AH And Constable S. 1996. Marine controlled-source electromagnetic sounding 1. Modelling and experimental data. Jour Geophys Res 101: 5507-5517.

Goldman M. 1990. Non-Conventional Methods in Geoelectrical Prospecting. New York: Ellis Horwood, 153 p. 
Guimarẽes LG And SAMPAio EES. 2008a. Debye analysis applied to multiple reflections and attenuation of electromagnetic plane waves in a stratified sea substratum. J Quant Spectrosc Radiat Transf 109: 811-821.

Guimarães LG And SAmpaio EES. 2008b. A note on Snell laws for electromagnetic plane waves in lossy media. J Quant Spectrosc Radiat Transf 109: 2124-2140.

LøSETH LO AND URSIN B. 2007. Electromagnetic fields in planarly layered anisotropic media. Geophys Jour Internat 170: 44-80.

Papoulis A. 1962. The Fourier Integral and its Applications. San Francisco: McGraw-Hill Book Company, 318 p.

Pinheiro JCA And Sampaio EeS. 1993. The magnetic field of the magnetic tail. In: Proceedings of the Cannes Conference on Undersea Defence Technology, p. 498-501.

RAYNER J. 2007. Geophysicists at war: mines, magnetism and memories Part 2: the Australian connection. Preview 30: 32-34.

SAMPAIO EES AND FOKKEMA JT. 1992. Scattering of monochromatic acoustic and electromagnetic plane waves by two quarter spaces. Jour Geophys Res 97: 1953-1963.

SAMPAIO EES AND Popov MM. 1997. Zero-order time domain scattering of electromagnetic plane waves by two quarter spaces. Radio Science 32: 305-315.

SCHOLL C AND EDWARDS RN. 2007. Marine downhole to seafloor dipole-dipole electromagnetic methods and the resolution of resistive targets. Geophysics 72: WA39-WA49.

Sokolov I, Klafter J And Blumen A. 2002. Fractional kinetics. Physics Today 55: 48-54.

Sommerfeld A. 1949. Partial Differential Equation in Physics, vol. VI. London: Academic Press, 335 p.

Souza H AND Sampaio EES. 2001. Apparent resistivity and spectral induced polarization in the submarine environment. An Acad Bras Cienc 73: 429-444.

SPIES BR AND HABASHY TM. 1995. Sensitivity analysis of crosswell electromagnetics. Geophysics 60: 834-845.

StRATTON JA. 1941. Electromagnetic Theory. New York: McGraw-Hill Book Company, 615 p.

TORRes-Verdín C AND Bostick JR FX. 1992. Implications of the Born approximation for the magnetotelluric problem in three-dimensional environment. Geophysics 57: 587-602.

TORRES-VERdín C AND HABASHY TM. 2001. Rapid numerical simulation of axisymmetric single-well induction data using the extended Born approximation. Radio Science 35: 1287-1306.

TSENG HW, LEE KH AND BECKER A. 2003. 3-D interpretation of electromagnetic data using a modified extension Born approsimation. Geophysics 68: 127-137.

WAIT JR. 1982. Geo-electromagnetism. New York: Academic Press Inc, 268 p.

WANNAMAKer PE, Hohmann GW AND SANFILIPo WA. 1984. Electromagnetic modeling of three-dimensional bodies in layered earths using integral equations. Geophysics 49: 60-74.

WARD SH AND HOHMANN GW. 1988. Electromagnetic theory for geophysical application. In: NABIGHIAN MN (Ed), Electromagnetic Methods in Applied Geophysics, vol. I, Theory, Chapter 3, Tulsa, SEG p. 130-311.

Wolfgram PA, Edwards RN, Law LK and Bone MN. 1986. Polymetallic sulfide exploration on the deep sea floor: The feasibility of the MINI-MOSES experiment. Geophysics 51: 1808-1818.

WYNN JS. 1988. Titanium geophysics - the application of induced polarization to sea-floor mineral exploration. Geophysics 53: 386-401. 


\section{APPENDIXES}

\section{A BINOMIAL EXPANSION OF THE KERNEL FUNCTIONS}

Take into account that $\left|e^{-2 \alpha_{1} h_{1}}\right|<1$ and $\left|R_{1, j}\right|<1, \quad j=0,2$, and express the reflection coefficient as,

$$
R_{1, j}=\frac{2 \alpha_{1}}{\alpha_{1}+\alpha_{j}}-1, j=0,2
$$

Next, expand the denominator of $F_{x, 1}^{ \pm}(\lambda, s)$ by the binomial theorem and neglect second and higher order terms to obtain:

$$
\begin{aligned}
\int_{\beta-i \infty}^{\beta+i \infty} \frac{F_{x, 1}^{+}(\lambda, s)}{s} e^{+\alpha_{1} z} e^{+s t} d s \approx & \int_{\beta-i \infty}^{\beta+i \infty}\left\{\left(\frac{2 \lambda}{s\left(\alpha_{1}+\alpha_{2}\right)}-\frac{\lambda}{s \alpha_{1}}\right) e^{-\alpha_{1} q_{1}}\right. \\
& \left.+\left(-\frac{2 \lambda}{s\left(\alpha_{1}+\alpha_{0}\right)}-\frac{2 \lambda}{s\left(\alpha_{1}+\alpha_{2}\right)}+\frac{\lambda}{s \alpha_{1}}\right) e^{-\alpha_{1} q_{2}}\right\} e^{+s t} d s, \\
\int_{\beta-i \infty}^{\beta+i \infty} \frac{F_{x, 1}^{-}(\lambda, s)}{s} e^{-\alpha_{1} z} e^{+s t} d s \approx & \int_{\beta-i \infty}^{\beta+i \infty}\left\{\left(\frac{2 \lambda}{s\left(\alpha_{1}+\alpha_{0}\right)}-\frac{\lambda}{s \alpha_{1}}\right) e^{-\alpha_{1} q_{3}}\right. \\
& \left.+\left(-\frac{2 \lambda}{s\left(\alpha_{1}+\alpha_{0}\right)}-\frac{2 \lambda}{s\left(\alpha_{1}+\alpha_{2}\right)}+\frac{\lambda}{s \alpha_{1}}\right) e^{-\alpha_{1} q_{4}}\right\} e^{+s t} d s .
\end{aligned}
$$

Equations A1 contain three types of Laplace transforms. The coefficients $q_{j}$ are positive, $j=1,2,3,4$, because

$$
q_{1}=2 h_{1}-z-z_{0} ; \quad q_{2}=2 h_{1}-z+z_{0} ; \quad q_{3}=z+z_{0} ; \quad q_{4}=2 h_{1}+z-z_{0} .
$$

When $z=h_{1}$,

$$
q_{1}=h_{1}-z_{0} ; \quad q_{2}=h_{1}+z_{0} ; \quad q_{3}=h_{1}+z_{0} ; \quad q_{4}=3 h_{1}-z_{0} .
$$

\section{B LAPLACE TRANSFORM OF THE FIRST ORDER TERMS}

Substitute in Equation A1 $s=p-\gamma, \gamma=\frac{\lambda^{2}}{\mu_{0} \sigma_{1}}$ and write:

$$
\alpha_{0}=\frac{\sqrt{\left(p-p_{0}\right)\left(p-\dot{p}_{0}\right)}}{c_{0}} ; \quad \alpha_{1}=\sqrt{\mu_{0} \sigma_{1}} \sqrt{p} ; \text { and } \alpha_{2}=\sqrt{\mu_{0} \sigma_{2}} \sqrt{p+p_{1}},
$$

where $p=0, p_{0}=\gamma+i c_{0} \lambda, \dot{p}_{0}=\gamma-i c_{0} \lambda$, and

$$
p_{1}=\frac{\lambda^{2}\left(\sigma_{1}-\sigma_{2}\right)}{\mu_{0} \sigma_{1} \sigma_{2}}=\frac{\gamma\left(\sigma_{1}-\sigma_{2}\right)}{\sigma_{2}}
$$

represent the four branch points. The residue at the pole $p=+\gamma$ vanishes. The vertical straight line in Figure B1 represents the Bromwich path. We shall evaluate the integrals modifying it to a closed circuit (Papoulis 1962).

The three types of Laplace transforms of Equation A1 yield, respectively, the following expressions (Abramowitz and Stegun 1968): 


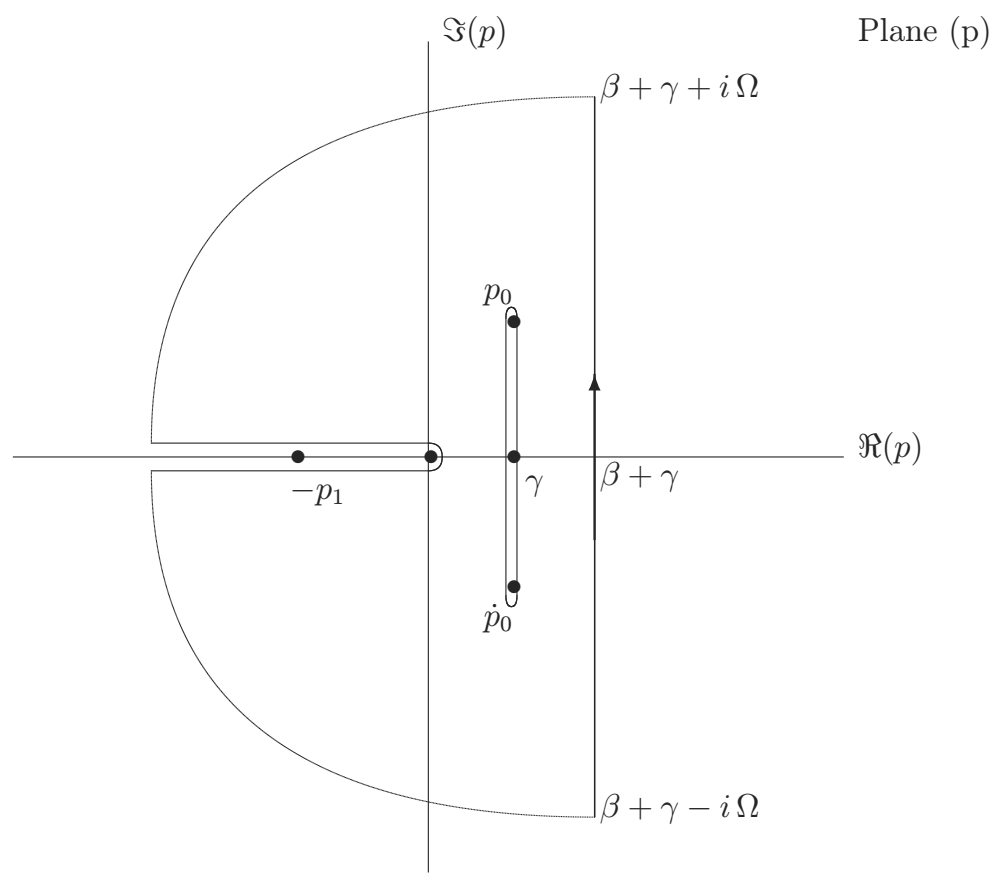

Fig. B1 - Distortion of the Bromwich path $(\Re(p)=\beta+\gamma)$ into a closed contour for the integration of Equations B1, B2, and B4. The function is analytical inside the contour. The origin, $-p_{1}, p_{0}$, and $\dot{p}_{0}$ are branch points, and $\gamma$ is the pole.

\section{FIRST TRANSFORM}

$$
\begin{aligned}
& \lambda \int_{B r} \frac{e^{-\alpha_{1} q_{j}} e^{s t}}{s \alpha_{1}} d s=\frac{e^{-\gamma t}}{2} \int_{B r}\left(\frac{1}{\sqrt{p}(-\sqrt{\gamma}+\sqrt{p})}-\frac{1}{\sqrt{p}(+\sqrt{\gamma}+\sqrt{p})}\right) e^{-\sqrt{\mu_{0} \sigma_{1}} q_{j} \sqrt{p}} e^{+p t} d p, \\
& \sum_{j=1}^{4} \frac{(-1)^{j} \lambda}{2 \pi i} \int_{B r} \frac{e^{-\alpha_{1} q_{j}} e^{s t}}{s \alpha_{1}} d s=\sum_{j=1}^{4}(-1)^{j}\left(\frac{e^{-\lambda q_{j}}}{2} \operatorname{erfc}\left(-\lambda \chi+\frac{q_{j}}{2 \chi}\right)\right. \\
& \left.-\frac{e^{+\lambda q_{j}}}{2} \operatorname{erfc}\left(+\lambda \chi+\frac{q_{j}}{2 \chi}\right)\right) u(t)
\end{aligned}
$$

where:

$$
\chi=\sqrt{\frac{t}{\mu_{0} \sigma_{1}}} .
$$

The circuit of the two integrals of Equation B1 is represented in Figure B1 with the following parts: the semi-circle $\Omega \rightarrow \infty$, for $\Re(p)<\beta+\gamma$; the circle $\epsilon \rightarrow 0$ around the branch point $p=0$; the contour of the branch cut $(-\infty \rightarrow 0)$; and the circle around the pole $p=+\gamma$. The two terms of Equation B1 correspond to the contour of the branch cut. The integrals along the circle $\epsilon \rightarrow 0$ around the branch point $p=0$ and along the semi-circle $\Omega \rightarrow \infty$ for $\Re(p)<\beta+\gamma$ vanish.

\section{SECOND TRANSFORM}

$$
\int_{B r} \frac{e^{-\alpha_{1} q_{j}} e^{s t}}{s\left(\alpha_{1}+\alpha_{2}\right)} d s=e^{-\gamma t} \int_{B_{r}} \frac{\left(\sqrt{\sigma_{1}} \sqrt{p}-\sqrt{\sigma_{2}} \sqrt{p+p_{1}}\right) e^{-\sqrt{\mu_{0} \sigma_{1}} q_{j} \sqrt{p}} e^{+p t}}{\sqrt{\mu_{0}}\left(\sigma_{1}-\sigma_{2}\right)(p-\gamma)^{2}} d p
$$




$$
\sum_{j=1}^{4, j \neq 3} \frac{(-1)^{j+1} \lambda}{\pi i} \int_{B r} \frac{e^{-\alpha_{1} q_{j}} e^{s t}}{s\left(\alpha_{1}+\alpha_{2}\right)} d s=\sum_{j=1}^{4, j \neq 3}(-1)^{j+1} g_{j}^{+}(\lambda, t) u(t) .
$$

The circuit of the two integrals of Equation B2 is represented in Figure B1, and is constituted of the following parts: the semi-circle $\Omega \rightarrow \infty$, for $\Re(p)<\beta+\gamma$; the circles $\epsilon \rightarrow 0$ around the branch points $p=0$ and $p=-p_{1}$; the contours of the branch cuts: $(-\infty \rightarrow 0)$ and from $-p_{1}$ to 0 ; and the circle around $p=+\gamma$. In Equation B2, the integrals along the circles with radius $\epsilon \rightarrow 0$ around the branch points $p=0$ and $p=-p_{1}$ and around $p=\Omega \rightarrow \infty$ vanish, and

$$
\begin{aligned}
\frac{\partial g_{j}^{+}(\lambda, t)}{\partial t}= & \frac{2 \lambda e^{-\gamma t} \sqrt{\sigma_{1}}}{\sqrt{\mu_{0}}\left(\sigma_{1}-\sigma_{2}\right)}\left\{\frac{1}{\sqrt{\pi t}} e^{-\frac{q_{j}^{2}}{4 \chi^{2}}}+\frac{\lambda \chi e^{+\lambda^{2} \chi^{2}}}{2 \sqrt{t}}\right. \\
& \times\left\{e^{-\lambda q_{j}} \operatorname{erfc}\left(-\lambda \chi+\frac{q_{j}}{2 \chi}\right)-e^{+\lambda q_{j}} \operatorname{erfc}\left(\lambda \chi+\frac{q_{j}}{2 \chi}\right)\right\} \\
& \left.+\frac{\sqrt{\sigma_{2}}\left(\sigma_{1}-\sigma_{2}\right) \sqrt{p_{1}}}{\pi \sqrt{\sigma_{1}}} \int_{0}^{1} \frac{N^{+}(p)}{\left(\sigma_{1}-\sigma_{2}\right) p+\sigma_{2}} e^{-p_{1} t p} d p\right\}, \\
& N^{+}(p)=\sqrt{1-p} \sin \left(\sqrt{\mu_{0} \sigma_{1} p_{1}} q_{j} \sqrt{p}\right) .
\end{aligned}
$$

THIRD TRANSFORM

$$
\begin{aligned}
& \int_{B r} \frac{e^{-\alpha_{1} q_{j}} e^{s t}}{S\left(\alpha_{1}+\alpha_{0}\right)} d s= \frac{e^{-\gamma t}}{\mu_{0} \epsilon_{0}} \int_{B r} \frac{\sqrt{\mu_{0} \sigma_{1}} \sqrt{p} e^{-\sqrt{\mu_{0} \sigma_{1}} q_{j} \sqrt{p}} e^{+p t}}{(p-\gamma)^{2}\left(\gamma+\frac{\sigma_{1}}{\epsilon_{0}}-p\right)} d p \\
&-\frac{e^{-\gamma t}}{\mu_{0} \epsilon_{0}} \int_{B r} \frac{\sqrt{\left(p-p_{0}\right)\left(p-\dot{p}_{0}\right)} e^{-\sqrt{\mu_{0} \sigma_{1}} q_{j} \sqrt{p}} e^{+p t}}{c_{0}(p-\gamma)^{2}\left(\gamma+\frac{\sigma_{1}}{\epsilon_{0}}-p\right)} d p, \\
& \sum_{j=2}^{4} \frac{(-1)^{j+1} \lambda}{\pi i} \int_{B r} \frac{e^{-\alpha_{1} q_{j}} e^{s t}}{s\left(\alpha_{1}+\alpha_{0}\right)} d s=\sum_{j=2}^{4}(-1)^{j+1}\left\{g_{j}^{-}(\lambda, t)+h_{j}^{-}(\lambda, t)\right\} u(t) .
\end{aligned}
$$

The circuit of the two integrals of Equation B4 is represented in Figure B1, and is constituted of the following parts: the semi-circle $\Omega \rightarrow \infty$, for $\Re(p)<\beta+\gamma$; the circles $\epsilon \rightarrow 0$ around the branch points $p=0, p=p_{0}$ and $p=\dot{p}_{0}$ and of the pole $p=+\gamma$; and the contours of the two branch cuts: from $\dot{p}_{0}$ to $p_{0}$ and from $-\infty$ to 0 . The pole $p=\gamma+\frac{\sigma_{1}}{\epsilon_{0}}$ is not shown, and its two residues cancel out. In Equation B4 the integrals along the circles with radius $\epsilon \rightarrow 0$ around the branch points $p=0, p=p_{0}$ and $p=\dot{p}_{0}$, as well as around $p=\Omega \rightarrow \infty$, vanish. The term $g_{j}^{-}(\lambda, t)$ on the right-hand side of Equation B4 corresponds to the contour of the branch cut from $-\infty$ to 0 , and the term $h_{j}^{-}(\lambda, t)$ to the 
contour around the branch cut parallel to the imaginary axis, such that

$$
\begin{aligned}
& \frac{\partial g_{j}^{-}(\lambda, t)}{\partial t}=\frac{2 \lambda e^{-\gamma t}}{\sqrt{\mu_{0} \sigma_{1}}}\left\{\frac{\lambda \chi e^{+\lambda^{2} \chi^{2}}}{2 \sqrt{t}}\left\{e^{-\lambda q_{j}} \operatorname{erfc}\left(-\lambda \chi+\frac{q_{j}}{2 \chi}\right)-e^{+\lambda q_{j}} \operatorname{erfc}\left(\lambda \chi+\frac{q_{j}}{2 \chi}\right)\right\}\right. \\
& -\frac{\sqrt{\gamma+\frac{\sigma_{1}}{\epsilon_{0}}} e^{+\gamma t+\frac{\sigma_{1}}{\epsilon_{0}} t}}{2}\left\{e^{-\sqrt{\gamma+\frac{\sigma_{1}}{\epsilon_{0}}} \sqrt{\mu_{0} \sigma_{1}} q_{j}} \operatorname{erfc}\left(-\sqrt{\left(\gamma+\frac{\sigma_{1}}{\epsilon_{0}}\right) t}+\frac{q_{j}}{2 \chi}\right)\right. \\
& \left.\left.-e^{+\sqrt{\gamma+\frac{\sigma_{1}}{\epsilon_{0}}} \sqrt{\mu_{0} \sigma_{1}} q_{j}} \operatorname{erfc}\left(+\sqrt{\left(\gamma+\frac{\sigma_{1}}{\epsilon_{0}}\right) t}+\frac{q_{j}}{2 \chi}\right)\right\}\right\} \\
& +\frac{2 c_{0} \lambda e^{-\gamma t}}{\pi} \int_{0}^{+\infty} \frac{N^{-} e^{-t p}}{(p+\gamma)\left(p+\gamma+\frac{\sigma_{1}}{\epsilon_{0}}\right)} d p \\
& N^{-}(p)=\sqrt{\left(p+p_{0}\right)\left(p+\dot{p}_{0}\right)} \sin \left(\sqrt{\mu_{0} \sigma_{1}} q_{j} \sqrt{p}\right) . \\
& \frac{\partial h_{j}^{-}(\lambda, t)}{\partial t}=-\frac{4 c_{0} \lambda}{\pi} \int_{0}^{c_{0} \lambda}\left\{\frac{\sigma_{1}}{\epsilon_{0}} \frac{\sin \left(p t-b_{j}\right)}{p}+\cos \left(p t-b_{j}\right)\right\} \frac{\sqrt{c_{0}^{2} \lambda^{2}-p^{2}} e^{-a_{j}}}{\frac{\sigma_{1}^{2}}{\epsilon_{0}^{2}}+p^{2}} d p, \\
& a_{j}=\sqrt{\frac{\mu_{0} \sigma_{1}}{2}} \sqrt{\sqrt{\gamma^{2}+p^{2}}+\gamma} q_{j}, \\
& b_{j}=\sqrt{\frac{\mu_{0} \sigma_{1}}{2}} \sqrt{\sqrt{\gamma^{2}+p^{2}}-\gamma} q_{j} .
\end{aligned}
$$

The first two terms on the right-hand side of Equation 15 yield the following Hankel transforms:

$$
\begin{aligned}
(\mathcal{T H})_{1} & =-\left(\frac{\partial}{\partial y}\right) \int_{0}^{+\infty} \lambda e^{-\lambda^{2} \chi^{2}} J_{0}(\lambda r) \\
d \lambda, & =+\left(\frac{y}{r}\right) \int_{0}^{+\infty} \lambda^{2} e^{-\lambda^{2} \chi^{2}} J_{1}(\lambda r) d \lambda .
\end{aligned}
$$

Employing the tables of (Erdélyi 1954) we obtain the following result:

$$
\begin{aligned}
(\mathcal{T H})_{1} & =-\left(\frac{1}{2 \chi^{2}}\right) \Gamma(1)\left(\frac{\partial}{\partial y}{ }_{1} F_{1}\left(1 ; 1 ;-\left(\frac{r}{2 \chi}\right)^{2}\right)\right) \\
& =+\left(\frac{y}{4 \chi^{4}}\right)(0 !) e^{-\left(\frac{r}{2 \chi}\right)^{2}} L_{0}\left(\left(\frac{r}{2 \chi}\right)^{2}\right)
\end{aligned}
$$

Employing the properties of the $\Gamma(\eta)$ function, of Kummer's hypergeometric series, and of the generalized Laguerre polinomium on Equation C2, we conclude that

$$
(\mathcal{T H})_{1}=\left(\frac{y}{4 \chi^{4}}\right) e^{-\left(\frac{r}{2 \chi}\right)^{2}}
$$


and therefore:

$$
\begin{array}{r}
100 y \int_{-\frac{L}{2}}^{+\frac{L}{2}}\left\{\int_{0}^{+\infty}\left\{\sum_{j=1}^{4}(-1)^{j} e^{-\left(\frac{q_{j}}{2 \chi}\right)^{2}} \frac{\lambda^{2} e^{-\lambda^{2} \chi^{2}}}{\sqrt{\pi} \mu_{0} \sigma_{1} \chi}\right\} J_{1}(\lambda r) d \lambda\right\} \frac{d x_{0}}{r} \\
=\frac{\pi \sigma_{1} y e^{\left(-\frac{y}{2 \chi}\right)^{2}}}{10^{5} t^{2}}\left\{\operatorname{erf}\left(\frac{x+\frac{L}{2}}{2 \chi}\right)-\operatorname{erf}\left(\frac{x-\frac{L}{2}}{2 \chi}\right)\right\}\left(\sum_{j=1}^{4}(-1)^{j} e^{-\left(\frac{q_{j}}{2 \chi}\right)^{2}}\right), \\
100 y \int_{-\frac{L}{2}}^{+\frac{L}{2}}\left\{\int_{0}^{+\infty}\left\{\sum_{j=1}^{4, j \neq 3}(-1)^{j+1} e^{-\left(\frac{q_{j}}{2 \chi}\right)^{2}} \frac{2 \lambda^{2} e^{-\lambda^{2} \chi^{2}}}{\sqrt{\pi} \mu_{0}\left(\sigma_{1}-\sigma_{2}\right) \chi}\right\} J_{1}(\lambda r) d \lambda\right\} \frac{d x_{0}}{r} \\
=\frac{2 \pi \sigma_{1}^{2} y e^{\left(-\frac{y}{2 \chi}\right)^{2}}}{10^{5}\left(\sigma_{1}-\sigma_{2}\right) t^{2}}\left\{\operatorname{erf}\left(\frac{x+\frac{L}{2}}{2 \chi}\right)-\operatorname{erf}\left(\frac{x-\frac{L}{2}}{2 \chi}\right)\right\}\left(\sum_{j=1}^{4, j \neq 3}(-1)^{j+1} e^{-\left(\frac{q_{j}}{2 \chi}\right)^{2}}\right) .
\end{array}
$$

\section{SECOND HANKEL TRANSFORM}

The two first terms inside the brackets on the right-hand side of Equation 16, combined with Equation 13, yield the following Hankel transform:

$$
\begin{aligned}
(\mathcal{T H})_{2} & =+\left(4-\frac{\sigma_{2}}{\sigma_{1}}\right)\left(\frac{\partial}{\partial y}\right) \int_{0}^{+\infty} \lambda^{2} e^{-\lambda^{2} \chi^{2}} J_{0}(\lambda r) d \lambda \\
& =-\left(4-\frac{\sigma_{2}}{\sigma_{1}}\right)\left(\frac{y}{r}\right) \int_{0}^{+\infty} \lambda^{3} e^{-\lambda^{2} \chi^{2}} J_{1}(\lambda r) d \lambda
\end{aligned}
$$

By employing the tables of (Erdélyi 1954) we obtain the following result:

$$
\begin{aligned}
(\mathcal{T H})_{2} & =+\left(4-\frac{\sigma_{2}}{\sigma_{1}}\right)\left(\frac{1}{2 \chi^{3}}\right) \Gamma\left(\frac{3}{2}\right)\left(\frac{\partial}{\partial y}{ }_{1} F_{1}\left(\frac{3}{2} ; 1 ;-\left(\frac{r}{2 \chi}\right)^{2}\right)\right), \\
& =-\left(4-\frac{\sigma_{2}}{\sigma_{1}}\right)\left(\frac{y}{4 \chi^{5}}\right)\left(\frac{1}{2} !\right) e^{-\left(\frac{r}{2 \chi}\right)^{2}} L_{\frac{1}{2}}^{1}\left(\left(\frac{r}{2 \chi}\right)^{2}\right) .
\end{aligned}
$$

We shall prove that the two forms in Equation D2 give the same result. First consider the following properties of $\Gamma(\eta): \Gamma\left(\frac{1}{2}\right)=\sqrt{\pi} ; \Gamma(\eta+1)=\eta \Gamma(\eta)=\eta !$; and

$$
\Gamma\left(n+\frac{1}{2}\right)=\frac{\Pi_{k=0}^{n-1}(2 n+1)}{2^{n}} \Gamma\left(\frac{1}{2}\right) .
$$

Employing the general expression of Kummer's confluent hypergeometric series,

$$
\begin{aligned}
& { }_{1} F_{1}\left(a ; b ;-\left(\frac{r}{2 \chi}\right)^{2}\right)=\sum_{n=0}^{\infty} \frac{(-1)^{n} \Gamma(a+n) / \Gamma(a)}{(\Gamma(b+n) / \Gamma(b)) n !}\left(\frac{r^{2}}{4 \chi^{2}}\right)^{n}, \\
& { }_{1} F_{1}\left(\frac{3}{2} ; 1 ;-\left(\frac{r}{2 \chi}\right)^{2}\right)=\sum_{n=0}^{\infty} \frac{(-1)^{n}\left(\Pi_{k=0}^{n}(2 k+1)\right)}{2^{n}(n !)^{2}}\left(\frac{r^{2}}{4 \chi^{2}}\right)^{n},
\end{aligned}
$$


we obtain:

$$
\frac{\partial}{\partial y}{ }_{1} F_{1}\left(\frac{3}{2} ; 1 ;-\left(\frac{r}{2 \chi}\right)^{2}\right)=\left(\frac{2 y}{r^{2}}\right) \sum_{n=1}^{\infty} \frac{n(-1)^{n}\left(\Pi_{k=0}^{n}(2 k+1)\right)}{2^{n}(n !)^{2}}\left(\frac{r^{2}}{4 \chi^{2}}\right)^{n} .
$$

Also taking into account the representation of Laguerre's generalized polynomial of order $\eta$ and coefficient $\alpha$

we obtain

$$
L_{\eta}^{\alpha}(\chi)=\left(\frac{e^{\chi} \chi^{-\alpha}}{\eta !}\right)\left(\frac{d^{\eta}}{d \chi^{\eta}}\left(e^{-\chi} \chi^{\eta+\alpha}\right)\right)
$$

$$
L_{\frac{1}{2}}^{1}(\chi)=\left(\frac{e^{\chi} \chi^{-1}}{\left(\frac{1}{2} !\right)}\right)\left(\frac{d^{\frac{1}{2}}}{d \chi^{\frac{1}{2}}}\left(e^{-\chi} \chi^{\frac{3}{2}}\right)\right) .
$$

According to (Sokolov et al. 2002)

$$
\frac{d^{\frac{1}{2}} \chi^{\mu}}{d \chi^{\frac{1}{2}}}=\frac{\Gamma(\mu+1)}{\Gamma\left(\mu+\frac{1}{2}\right)} \chi^{\mu-\frac{1}{2}}, \quad \mu>-1 .
$$

Applying fractional derivative to the Taylor's series expansion of the exponential function, we conclude that

$$
\left(\frac{d^{\frac{1}{2}}}{d \chi^{\frac{1}{2}}}\left(e^{-\chi} \chi^{\frac{3}{2}}\right)\right)=\sum_{p=0}^{\infty} \frac{(-1)^{p}}{(p) !} \frac{\Gamma\left(p+\frac{5}{2}\right)}{\Gamma(p+2)} \chi^{p+1}
$$

where: $\Gamma(p+2)=(p+1)$ ! and

$$
\Gamma\left(p+\frac{5}{2}\right)=\Gamma\left((p+2)+\frac{1}{2}\right)=\frac{\left(\Pi_{k=0}^{p}(2 k+3)\right)}{2^{p+2}} \Gamma\left(\frac{1}{2}\right) .
$$

Therefore, we may rewrite Equation D2 as:

$$
(\mathcal{T H})_{2}=\left(4-\frac{\sigma_{2}}{\sigma_{1}}\right) \frac{\sqrt{\pi} y}{2 \chi^{3} r^{2}} \sum_{n=1}^{\infty} \frac{n(-1)^{n}\left(\Pi_{k=0}^{n}(2 k+1)\right)}{2^{n}(n !)^{2}}\left(\frac{r}{2 \chi}\right)^{2 n} .
$$

Substituting Equations D3 and C3 in Equation 13, we obtain the following expression for $b_{z s}$ in nanotesla:

$$
\begin{aligned}
b_{z s}(R, t)= & \frac{25 y}{\sqrt{\pi} \mu_{0} \sigma_{1} \chi^{3}}\left\{2 \pi\left(4-\frac{\sigma_{2}}{\sigma_{1}}\right) \sum_{n=1}^{\infty} \frac{n(-1)^{n}\left(\Pi_{k=1}^{n}(2 k+1)\right) y^{2 n-1}}{2^{3 n}(n !)^{2} \chi^{2 n}}\right. \\
& \sum_{j=1}^{n} \frac{(n-1) !}{(2 j-1)(j-1) !(n-j) !}\left(\left(\frac{x+\frac{L}{2}}{y}\right)^{2 j-1}-\left(\frac{x-\frac{L}{2}}{y}\right)^{2 j-1}\right) \\
& \left.+\frac{\sqrt{\pi} e^{-\left(\frac{y}{2 \chi}\right)^{2}}\left(e^{-\left(\frac{z-z_{0}}{2 \chi}\right)^{2}}+e^{-\left(\frac{z+z_{0}}{2 \chi}\right)^{2}}\right)}{\chi}\left(\operatorname{erf}\left(\frac{x+\frac{L}{2}}{2 \chi}\right)-\operatorname{erf}\left(\frac{x-\frac{L}{2}}{2 \chi}\right)\right)\right\} .
\end{aligned}
$$

The calculus of the double series of Equation D4 becomes laborious for high values of the argument. So, it is necessary to analyze its asymptotic behavior when $y$ and $t$ are such that $\frac{y}{\chi}>>1$. The fact that

$$
\lim _{\eta \rightarrow-\infty}{ }_{1} F_{1}\left(\frac{3}{2} ; 1 ; \eta\right)=\frac{\Gamma(1)}{\Gamma\left(-\frac{1}{2}\right)}(-\eta)^{-\frac{3}{2}}
$$


implies that

$$
\begin{gathered}
\lim _{\left(\frac{r}{2 \chi}\right)^{2} \rightarrow \infty}{ }_{1} F_{1}\left(\frac{3}{2} ; 1 ;-\left(\frac{r}{2 \chi}\right)^{2}\right)=-\frac{4 \chi^{3}}{\sqrt{\pi} r^{3}}, \\
\lim _{\left(\frac{r}{2 \chi}\right)^{2} \rightarrow \infty}\left(\frac{\partial}{\partial y}{ }_{1} F_{1}\left(\frac{3}{2} ; 1 ;-\left(\frac{r}{2 \chi}\right)^{2}\right)\right)=\frac{12 y \chi^{3}}{\sqrt{\pi} r^{5}}
\end{gathered}
$$

and consequently we have:

$$
\frac{12 y \chi^{3}}{\sqrt{\pi}} \int_{-\frac{L}{2}}^{+\frac{L}{2}} \frac{d x_{0}}{r^{5}}=\frac{4 \chi^{3}}{\sqrt{\pi} y^{3}}\left\{\frac{(x+L / 2)\left(3 y^{2}+2(x+L / 2)^{2}\right)}{\left(y^{2}+(x+L / 2)^{2}\right)^{3 / 2}}-\frac{(x-L / 2)\left(3 y^{2}+2(x-L / 2)^{2}\right)}{\left(y^{2}+(x-L / 2)^{2}\right)^{3 / 2}}\right\} .
$$

